Japan. J. Math.

Vol. 7, No. 2, 1981

\title{
Sur la classe caractéristique exotique de Lazarov-Pasternack en codimension 2, II.
}

\author{
By Kenji Yamato*)
}

(Received September 25, 1980)

\section{Introduction}

Nous considérons les feuilletages riemanniens de codimension 2 sur une variété $M$ dont les fibrés normaux sont trivials. Pour un tel feuilletage $\mathscr{F}$, C. Lazarov et J. Pasternack ([12]) ont construit une classe caractéristique exotique $L P(\mathscr{F})$ dans $H^{3}(M ; R)$.

Dans le premier article [17], nous avons considéré des certaines relations entre la classe caractéristique exotique $L P(\mathscr{F})$ et les propriétés qualitatives de $\mathscr{F}$.

Le but de cet article est de trouver une interprétation géométrique de la classe $L P(\mathscr{F})$ dans un cas simple où la variété $M$ est orientable fermée et de dimension 3. Notre résultat principal est le théorème suivant:

THÉORÈme 1. Dans les hypothèses ci-dessus, si le premier nombre de Betti de la variété $M$ n'est pas 1 , les deux conditions sont équivalentes:

(1) la classe de Lazarov-Pasternack LP(F) est nulle;

(2) il existe un feuilletage $\mathscr{H}$ de codimension 1 dont les feuilles sont des réunions de feuilles de $\mathscr{F}$.

On peut trouver une autre interprétation de la classe $L P(\mathscr{F})$ dans le paragraphe 1 (voir REMARQUE 1).

En général, il est facile de voir que la condition (2) implique la condition (1) (voir [17], nous disons dans le cas (2) que le feuilletage $\mathscr{F}$ admet une 1extension $\mathscr{H})$. Le probléme est donc de démontrer que la condition (1) implique la condition (2). Nous allons démontrer le théorème 1 dans chaque cas du théorème de $\mathrm{P}$. Molino ([14]) qui classifie les feuilletages riemanniens de codimension 2 sur une variété fermée.

Nous rappelons dans le paragraphe 1 la définition de la classe de LazarovPasternack suivant [12] et le théoréme de Molino. Les résultats correspondants aux cas du théorème de Molino apparaissent aussi dans ce paragraphe.

*) Supported by Grant-in-Aid for Encouragement of Young Scientist. 
Dans des cas du théorème de Molino où le feuilletage $\mathscr{F}$ ne possède pas de feuille compacte, on peut voir qu'il admet une 1-extension en dimension 3 et la classe $L P(\mathscr{F})$ est donc nulle, en utilisant un théorème de R. Blumenthal ([2]). La démonstration sera donnée dans le paragraphe 6.

Dans les autres cas, nous introduisons dans le paragraphe 2 "la classe localisée"" de la classe $L P(\mathscr{F})$ lorsqu'une 1-extension singulière du feuilletage $\mathscr{F}$ est donnée (cf. [1]). Une interprétation géométrique de la classe localisée est considérée dans le paragraphe 3 , et elle est utilisée dans les paragraphes 4 et 5 pour savoir si le nombre de Lazarov-Pasternack $L P(\mathscr{F})([M])$ est zéro ou non-zéro. Par exemple, nous considérons dans le paragraphe 4 le cas où toutes les feuilles sont compactes. Les feuilles sont alors les orbites d'une $S^{1}$-action dont l'espace d'orbites est une surface orientable fermée et de genre g. L'application immédiate de l'interprétation géométrique implique que le nombre $L P(\mathscr{F})([M])$ est non-zéro si $g \geqq 1$ et $\mathscr{F}$ n'est pas sans holonomie. Les cas exceptionnels où la classe $L P(\mathscr{F})$ est nulle mais le feuilletage n'admet pas de 1-extension, ils apparaissent seulement dans le cas précédent, lorsque $g=0$ et le premier nombre de Betti de $M$ est 1 .

Nous calculons explicitement dans le dernier paragraphe 7 les nombres de Lazarov-Pasternack des feuilletages riemanniens de codimension 2 sur $S^{3}$ qui étaient donnés dans [12] pour montrer que ces nombres sont variables continuellement.

Dans cet article, les variétés, les feuilletages, les fonctions, les formes différentielles et les champs de vecteurs etc..., sont toujours différentiables de classe $C^{\infty}$.

\section{§1. Préliminaires et résultats}

Soit $\mathscr{F}$ un feuilletage de codimension 2 sur une variété $M$. On dit qu'il est riemannien s'il admet une structure transversalement riemannienne $\tau$ :

$$
\tau=\left(\left\{\left(U_{\alpha}, f_{\alpha}\right)\right\},\left\{\gamma_{\alpha \beta}\right\},\left\{\left(\boldsymbol{R}_{\alpha}^{2}, g_{\alpha}\right)\right\}\right) ;
$$

(i ) $\left\{U_{\alpha}\right\}$ est un recouvrement ouvert de $M$;

(ii) $f_{\alpha}: U_{\alpha} \rightarrow R_{\alpha}^{2}$ est une submersion;

(iii) $g_{\alpha}$ est une métrique riemannienne sur $\boldsymbol{R}_{\alpha}^{2}$;

(iv) $f_{\alpha}=\gamma_{\alpha \beta} \circ f_{\beta}$ sur $U_{\alpha} \cap U_{\beta}$, où les applications

$$
\gamma_{\alpha \beta}:\left(\boldsymbol{R}_{\beta}^{2}, g_{\beta}\right) \longrightarrow\left(\boldsymbol{R}_{\alpha}^{2}, g_{\alpha}\right)
$$

sont des isométries locaux.

On supposera dans la suite que ce feuilletage $\mathscr{F}$ est riemannien et le fibré normal $\nu(\mathscr{F})$ est trivial. 
Nous rappelons comment la classe de Lazarov-Pasternack $L P(\mathscr{F})$ est définie pour ce feuilletage $\mathscr{F}$.

Sur le fibré normal $\nu(\mathscr{F})$, il existe une métrique quasi-fibrée $g$ définie localement par

$$
g=f_{\alpha}^{*} g_{\alpha} .
$$

Désignons par $\boldsymbol{P}$ (resp. $\boldsymbol{P}_{\alpha}$ ) le fibré principal des repères de $\nu(\mathscr{F})\left(\right.$ resp. $\left.\tau\left(\boldsymbol{R}_{\alpha}^{2}\right)\right)$ orthonormées pour la métrique $g$ (resp. $g_{\alpha}$ ) et par $\bar{\omega}_{\alpha}$ l'unique connexion sans torsion sur $P_{\alpha}$ définie par la métrique $g_{\alpha}$. Alors il existe une unique connexion sans torsion $\bar{\omega}$ sur le fibré $P$ définie localement par

$$
\bar{\omega}=f_{\alpha}^{*} \bar{\omega}_{\alpha} .
$$

Les courbures de ces connexions satisfont donc

$$
\bar{\Omega}=f_{\alpha}^{*} \bar{\Omega}_{\alpha} .
$$

Et il existe une section globale $\sigma$ de $P$ par la trivialité de $\nu(\mathscr{F})$. On a donc une connexion plate $\omega_{0}$ sur $P$ définie par la section $\sigma$.

Soit $e \in I^{1}(S O(2))$ le polynôme de Pfaff normalisé:

$$
e(X)=-x / 2 \pi, \quad \text { pour } \quad X=\left(\begin{array}{rr}
0 & x \\
-x & 0
\end{array}\right) \in \mathfrak{S}_{\mathfrak{O}}(2) .
$$

Alors on peut identifier la 3 -forme différentielle fermée $e\left(\bar{\omega}-\omega_{0}\right) \wedge e(\bar{\Omega})$ avec une unique 3-forme différentielle fermée sur $M$. Et on sait que sa classe de cohomologie dans $H^{3}(M ; R)$ ne dépend pas du choix de la section $\sigma$ ni de celui de la structure transversalement riemannienne $\tau$ ([12]).

Nous désignons par $L P(\mathscr{F})$ cette classe de cohomologie:

$$
L P(\mathscr{F})=\left[e\left(\bar{\omega}-\omega_{0}\right) \wedge e(\bar{\Omega})\right] \in H^{3}(M ; R) .
$$

Maintenant, soit $\bar{\theta}=\left(\vec{\theta}_{1}, \bar{\theta}_{2}\right)$ la forme fondamentale de $P$. Désignons par $\theta_{1}, \theta_{2}, \omega$ les images réciproques par la section $\sigma$ de $\bar{\theta}_{1}, \bar{\theta}_{2}, \bar{\omega}$ respestivement. Alors le feuilletage $\mathscr{F}$ est définie par

$$
\tau(\mathscr{F})=\left\{\theta_{1}=\theta_{2}=0\right\}
$$

en plus, comme la connexion $\bar{\omega}$ est sans torsion, on a

$$
\left(\begin{array}{l}
d \theta_{1} \\
d \theta_{2}
\end{array}\right)=\left(\begin{array}{rr}
0 & \omega \\
-\omega & 0
\end{array}\right)\left(\begin{array}{l}
\theta_{1} \\
\theta_{2}
\end{array}\right) \text {, et } d \omega=f \theta_{2} \wedge \theta_{1}
$$

où $f$ est une fonction différentiable sur $M$. On sait que la classe $L P(\mathscr{F})$ est représentée par la 3 -forme différentielle fermée $\left(1 / 4 \pi^{2}\right) \omega \wedge d \omega$ : 


$$
L P(\mathscr{F})=\left[\left(1 / 4 \pi^{2}\right) \omega \wedge d \omega\right] \in H^{3}(M ; R) .
$$

Nous faisons la remarque que la classe originale de Lazarov-Pasternack désignée par $\delta_{\mathscr{F}, g, \mathscr{S}}\left(\left\{\chi h_{\chi}\right\}\right)$ est en relation suivante avec la classe $L P(\mathscr{F})$ précédente:

$$
\delta_{\mathscr{s}, g, y,}\left(\left\{\chi h_{\chi}\right\}\right)=4 \pi^{2} L P(\mathscr{F}) .
$$

DÉfintrion 1. Le feuilletage $\mathscr{F}$ est dit 1-extendable si et seulement si il existe un feuilletage $\mathscr{H}$ de codimension 1 tel que le fibré tangent $\tau(\mathscr{F})$ de $\mathscr{F}$ soit un sous-fibré de $\tau(\mathscr{H})$ ([3]). Le feuilletage $\mathscr{H}$ est appelé une 1-extension de $\mathscr{F}$.

Notre résultats principal est le théorème suivant:

THÉORÈME 1. Si la variété $M$ est orientable fermée et de dimension 3 dont le premier nombre de Betti n'est pas 1, les deux conditions sont équivalentes;

(1) la classe de Lazarov-Pasternack LP(F) est nulle;

(2) le feuilletage $\mathscr{F}$ est 1-extendable.

En général, on sait:

LEMme 1.1 ([17]). Si le feuilletage $\mathscr{F}$ est 1-extendable, la classe de LazarovPasternack LP(F) est nulle.

Un théorème de P. Molino ([14]) est fondamental dans la démonstration $\mathrm{du}$ théorème 1 , qui classifie les feuilletages riemanniens $\mathscr{F}$ de codimension 2 sur une variété fermée $M$ (où, on ne suppose pas de trivialité de $\nu(\mathscr{F})$ ). Nous le rappelons.

ThÉoRÈme (P. Molino). Pour ces feuilletages $\mathscr{F}$, on a l'une des possibilités suivantes:

(A) toutes les feuilles de $\mathscr{F}$ sont compactes;

(B) le nombre des feuilles compactes de $\mathscr{F}$ est 1 ou 2;

(C) $\mathscr{F}$ admet une 1-extension dont les feuilles sont les adhérences des feuilles de $\mathscr{F}$;

(D) toutes les feuilles de $\mathscr{F}$ sont partout denses dans $M$.

Correspondant aux cas du théorème de Molino, nous obtenons les théorèmes suivants, avec l'hypothèse:

$[\mathrm{H}] \mathscr{F}$ est un feuilletage riemannien de codimension 2 sur une variété orientable fermée $M$ de dimension 3 dont le fibré normal $\nu(\mathscr{F})$ est trivial.

THÉORÈme A. Supposons que le feuilletage $\mathscr{F}$ soit au cas $(\mathrm{A})$ et désignons par $n$ le nombre de feuilles ayant d'holonomie. Alors, les feuilles du feuilletage 
$\mathscr{F}$ sont les orbites d'une $S^{1}$-action effective dont les invariants (orientés) ([15], [16]) sont

$$
\left\{b ;(o, g, 0,0) ;\left(\alpha_{1}, \beta_{1}\right), \cdots,\left(\alpha_{n}, \beta_{n}\right)\right\}
$$

De plus,

(1) si $n$ est zéro, le premier nombre de Betti $b_{1}(M)$ n'est pas 1 et les conditions suivantes sont équivalentes;

(i ) $g=1$;

(ii) le feuilletage $\mathscr{F}$ est 1-extendable;

(iii) la classe de Lazarov-Pasternack LP(F) est nulle;

(2) si $n$ est positif, $b_{1}(M)$ est $2 g$ ou 1 , en plus,

(i ) si $b_{1}(M)=1, L P(\mathscr{F})=0$;

(ii) si $b_{1}(M)=2 g$,

$$
L P(\mathscr{F})([M])=\left\{-2(1-g)+\sum_{i=1}^{n}\left(1-1 / \alpha_{i}\right)\right\}^{2} /\left(b+\sum_{i=1}^{n} \beta_{i} / \alpha_{i}\right) \neq 0,
$$

où $[M]$ est la classe de l'orientation de $M$.

ThÉonème B. Si le feuilletage $\mathscr{F}$ est au cas $(\mathrm{B})$, la classe $L P(\mathscr{F})$ est nonnulle (donc $\mathscr{F}$ n'est pas 1-extendable) et le premier nombre de Betti $b_{1}(M)$ est zéro.

THÉonème D. Si le feuilletage $\mathscr{F}$ est au cas (D), il est 1-extendable (donc la classe $L P(\mathscr{F})$ est nulle).

Il est claire que le théorème 1 est une conséquence immédiate des théorème de Molino, lemme 1.1 et théorèmes ci-dessus.

Remarque 1. On peut aussi déduire des théorèmes ci-dessus que les deux conditions suivantes sont équivalentes dans les hypothèses $d u$ théorème 1:

(1) la classe de Lazarov-Pasternack $L P(\mathscr{F})$ est non-nulle;

(2) tous les feuilletages suffisamment proches de $\mathscr{F}$ possèdent une feuille compacte.

Pour obtenir ce résultat, il suffit d'appliquer, au cas (A) (1), un résultat de F. Fuller [5] (cf. Springer Lecture Notes in Math., 652 p. 244-245), et celui de M. Hirch [9] (Theorem 1.1) aux cas (A) (2) et (B).

Mais, on sait que la condition (1) n'implique pas en général l'existence d'une feuille compacte de $\mathscr{F}$ en dimension $5([17])$.

Pour finir ce paragraphe, nous remarquons que le voisinage feuilleté d'une feuille compacte d'un feuilletage riemannien est caractérisé par l'holonomie linéaire. 
Soit $\mathscr{F}$ un feuilletage riemannien de codimension 2 dont le fibré normal est trivial, ayant une feuille compacte $F$. Désignons l'holonomie linéaire de $F$ par

$$
D h: \pi_{1}(F) \longrightarrow S O(2),
$$

c'est-à-dire, si $h$ est l'homomorphisme de l'holonomie de $F, D h(a)$ est l'application tangente de $h(a)$ en origine de $\boldsymbol{R}^{2}$ pour $a \in \pi_{1}(F)$.

Si $\tilde{F}$ désine le revêtement universel de $F$, la projection du produit cartésien $\tilde{U}^{\prime}=\tilde{F} \times D^{2}$ sur le second facteur détermine un feuilletage $\tilde{\mathscr{F}}^{\prime}$ de codimension 2 et une action de $\pi_{1}(F)$ sur $\tilde{U}^{\prime}$ est définie par

$$
\alpha \cdot(\tilde{x}, z)=(\alpha(\tilde{x}), D h(\alpha)(z)) \quad \operatorname{pour}(\tilde{x}, z) \in \tilde{F} \times D^{2}=\tilde{U}^{\prime},
$$

où $\alpha \in \pi_{1}(F)$ (resp. $D h(\alpha) \in S O(2)$ ) s'identifie avec l'automorphisme de $\tilde{F}$ (resp. l'action naturelle sur un disque $D^{2}$ de dimension 2). Puisque le feuilletage $\tilde{F}^{\prime}$ est invariant par cette action, on obtient un feuilletage $\mathscr{F}^{\prime}$ sur une variété $U^{\prime}=\pi_{1}(F) \backslash \tilde{U}^{\prime}\left(\cong F \times D^{2}\right)$.

On dit qu'un sous-ensemble $V$ d'une variété feuilletée est saturé si $V$ est une réunion des feuilles.

On remarque qu'un isométrie $f$ ayant un point fixe est l'identité si l'application tangente $f_{*}$ est l'identité en ce point, alors par un théorème de Haefliger ([7] p. 382) on a:

LEMME 1.2. Il existe un difféomorphisme feuilleté de classe $C^{\infty}$ d'un voisinage tubulaire saturé de la feuille compacte $F$ du feuilletage $\mathscr{F}$ sur la variété $U^{\prime} \cong F \times D^{2}$ munie du feuilletage $\mathscr{F}^{\prime}$, qui est l'identité sur $F$.

\section{§2. Localisation de la classe $\operatorname{LP}(\mathscr{F})$}

Soit $\mathscr{F}$ un feuilletage riemannien de codimension 2 sur une variété $M$ dont le fibré normal $\nu(\mathscr{F})$ est trivial. Nous fixons dans la suite la structure transversalement riemannienne $\tau$ de $\mathscr{F}$ et la section globale $\sigma$ du fibré $P$ de repères orthonormés de $\nu(\mathscr{F})$.

Une connexion $\omega$ du fibré $P$ (resp. $\left.P\right|_{o}$ sur un ouvert $O$ ) est dite transversalement projetable (par rapport à $(\mathscr{F}, \tau)$ ), s'il existe une connexion $\omega_{\alpha}$ sur le fibré $P_{\alpha}$ pour chaque $\alpha$ telle que la connexion $\omega$ satisfasse

$$
\left.\omega=f_{\alpha}^{*} \omega_{\alpha}, \quad \text { sur }\left.P\right|_{U_{\alpha}}\left(=f_{\alpha}^{*} P_{\alpha}\right) \text { (resp. }\left.P\right|_{o \cap U_{\alpha}}\right) \text {, }
$$

où $\tau=\left(\left\{\left(U_{\alpha}, f_{\alpha}\right),\left\{\gamma_{\alpha \beta}\right\},\left\{\left(\boldsymbol{R}_{\alpha}^{2}, g_{\alpha}\right)\right\}\right)\right.$.

DÉfinition 2.1. Nous disons que le feuilletage $\mathscr{F}$ admet une 1-extension singulière $(\mathscr{H}, \Sigma)$ si et seulement si il existe un feuilletage singulier $\mathscr{H}$ de codimension 1 dont l'ensemble de points singuliers $\Sigma$ est une réunion finie 
de feuilles compactes de $\mathscr{F}$, satifaisant les conditions suivantes;

(1) $\operatorname{sur} M-\Sigma, \quad \tau(\mathscr{F}) \subset \tau(\mathscr{H})$;

(2) pour chaque feuille compacte $F$ de $\mathscr{F}$ contenu dans $\Sigma$, il existe un champ de vecteurs $X$ sur un voisinage $U$ de $F$ qui s'annule seulement sur $F$ et satifait

$$
\tau(\mathscr{H})=\tau(\mathscr{F}) \oplus \boldsymbol{R} \cdot X, \quad \operatorname{sur} U-F
$$

Nous supposons dans la suite que le feuilletage $\mathscr{F}$ admet une 1-extension singulière $(\mathscr{H}, \Sigma)$ avec $\Sigma=\bigcup_{i} F_{i}$, et définissons la classe locolisée de $L P(\mathscr{F})$ dans $H^{1}(\Sigma ; R)\left(=\oplus_{i} H^{1}\left(F_{i} ; R\right)\right)$.

Soient $\bar{\theta}_{1}, \bar{\theta}_{2}, \bar{\omega}$ les formes différentielles sur $P$ et $g$ la métrique quasifibrée de $\nu(\mathscr{F})$ mentionnées dans le paragraphe 1 .

Puisque $\tau(\mathscr{F})$ est un sous-fibré de $\tau(\mathscr{H})$ sur $M-\Sigma$, on obtient une décomposition orthogonalle de $\nu(\mathscr{F})$ pour la métrique $g$

$$
\nu(\mathscr{F})=\nu_{1} \oplus \nu_{2} \quad \operatorname{sur} M-\Sigma, \quad \text { où } \tau(\mathscr{H})=\tau(\mathscr{F}) \oplus \nu_{1} \quad \text { sur } M-\Sigma .
$$

Il est donc facile de voir qu'il existe une connexion plate $\bar{\omega}^{0}$ sur $\left.P\right|_{M-\Sigma}$ dont l'espace horizontale est l'image de l'application tangente d'une section localle $s=\left(s_{1}, s_{2}\right)$ de $P$ telle que $s_{i}$ est une section de $\nu_{i}$ pour $i=1,2$. Et on a facilement:

Lemme 2.1 ([17]). La connexsion $\bar{\omega}^{0}$ sur $\left.P\right|_{M-\Sigma}$ satisfait les conditions suivantes:

(1) $\bar{\omega}^{0}$ est plate;

(2) $\bar{\omega}^{0}$ est transversalement projetable;

(3) l'espace horizontale de $\bar{\omega}^{0}$ contient l'espace déterminé par $\bar{\theta}_{1}=\bar{\theta}_{2}=\bar{\omega}$ $=0$.

En plus, nous avons:

Lemme 2.2. Soient $\bar{\omega}^{0}$ la connexion précédante et $\bar{\omega}$ la connexion sur $P$. Alors il existe une connexion $\bar{\omega}^{\prime}$ sur $P$ satisfaisant les conditions suivantes:

(1) pour chaque $F_{i}$ de $\Sigma$, il existe deux voisinages tubulaires saturés $V_{i}$ et $U_{i}$ de $F_{i}$ tels que

$$
\bar{\omega}^{\prime}= \begin{cases}\bar{\omega}^{0}, & \text { sur }\left.P\right|_{M-\cup U_{i}} \\ \bar{\omega}, & \text { sur }\left.P\right|_{V_{i}}\end{cases}
$$

et $V_{i} \subset U_{i}, U_{i} \cap U_{j}=\phi(i \neq j)$;

(2) $\bar{\omega}^{\prime}$ est transversalement projetable;

(3) l'espace horizontale de $\bar{\omega}^{\prime}$ contient l'espace déterminé par $\bar{\theta}_{1}=\bar{\theta}_{2}=\bar{\omega}$ $=0$. 
DÉmonstration. Soit $U_{i}$ le voisinage tubulaire saturé de la feuille compacte $F_{i}$ satisfaisant le lemme 1.2. Alors, on peut avoir facilement une fonction différentiable

$$
r_{i}: U_{i}=F_{i} \times D^{2} \longrightarrow[0,1]
$$

possédant les propriétés suivantes:

(i) $r_{i}$ est constante le long des feuilles;

(ii) $r_{i} \equiv 0$ sur $F_{i}$ et $r_{i} \equiv 1$ sur le bord $F_{i} \times \partial D^{2}$ de $U_{i}$.

Prenons maintenant une fonction différentiable

$$
\lambda:[0,1] \longrightarrow[0,1]
$$

telle que $\lambda(t) \equiv 1$ pour $0 \leqq t<\varepsilon$ et $\lambda(t) \equiv 0$ pour $1-\varepsilon<t \leqq 1$. Alors la connexion $\bar{\omega}^{\prime}$ est définie par

$$
\begin{aligned}
\left(\bar{\omega}^{\prime}\right)_{u} & =\left(\lambda \circ r_{i} \circ \pi(u)\right) \cdot \bar{\omega}+\left(1-\lambda \circ r_{i} \circ \pi(u)\right) \cdot \bar{\omega}^{0} \quad \text { en }\left.u \in P\right|_{U_{i}} \\
\text { et } \quad \bar{\omega}^{\prime} & =\left.\bar{\omega}^{0} \quad \operatorname{sur} P\right|_{M-\cup U_{i}},
\end{aligned}
$$

où $\pi: P \rightarrow M$ est la projection.

Il est claire que la connexion $\bar{\omega}^{\prime}$ satisfait le point (1) du lemme. En plus, les deux connexions $\bar{\omega}$ et $\bar{\omega}^{0}$ possèdent les propriétés des points (2) et (3) du lemme et les fonctions $r_{i}$ sont constantes le long des feuilles. Par suite, $\bar{\omega}^{\prime}$ satisfait aussi les deux points (2) et (3).

c.q.f.d.

Le lemme suivant nous admet à localiser la classe $L P(\mathscr{F})$.

Lemme 2.3. Désignons par $\omega^{\prime}$ l'image réciproque de la connexion $\bar{\omega}^{\prime}$ de $P$ par la section $\sigma . \quad$ Alors la classe de Lazarov-Pasternack LP $(\mathscr{F})$ est représentée par la 3-forme différentielle fermée $\left(1 / 4 \pi^{2}\right) \omega^{\prime} \wedge d \omega^{\prime}$.

Démonstration. Par la définition, la classe $L P(\mathscr{F})$ est représentée par $\left(1 / 4 \pi^{2}\right) \omega \wedge d \omega$, où $\omega=\sigma^{*} \bar{\omega}$.

D'abord, on remarque

$$
\left(\omega^{\prime}-\omega\right) \wedge d \omega \equiv\left(\omega^{\prime}-\omega\right) \wedge d \omega^{\prime} \equiv 0,
$$

parce que les deux connexions $\bar{\omega}$ et $\bar{\omega}^{\prime}$ possèdent les propriété des points (2) et (3) du lemme 2.2. Alors on a

$$
\begin{aligned}
\omega^{\prime} \wedge d \omega^{\prime}-\omega \wedge d \omega & =\left(\omega^{\prime}-\omega\right) \wedge d \omega^{\prime}+\omega \wedge\left(d \omega^{\prime}-d \omega\right) \\
& =\omega \wedge d\left(\omega^{\prime}-\omega\right) \\
& =d\left(\left(\omega^{\prime}-\omega\right) \wedge \omega\right) .
\end{aligned}
$$

Soit

$$
f_{D^{2}}: A^{*}\left(U_{i}, \partial U_{i}\right) \longrightarrow A^{*-2}\left(F_{i}\right)
$$


l'intégration le long des fibres pour $U_{i}=F_{i} \times D^{2}$, qui induit un isomorphisme en cohomologie ([6]).

La connexion $\bar{\omega}^{\prime}$ est plate sur $\left.P\right|_{M-\cup U_{i}}$ par les lemmes 2.1 (1) et 2.2 (1), donc $d \omega^{\prime} \equiv 0$ sur $M-\cup U_{i}$. Par suit, on a

$$
\omega^{\prime} \wedge d \omega^{\prime} \in A^{3}\left(M, M-\cup U_{i}\right) \text { et }\left.\left(\omega^{\prime} \wedge d \omega^{\prime}\right)\right|_{U_{i}} \in A^{3}\left(U_{i}, \partial U_{i}\right) .
$$

En vertu du lemme 2.3 , la classe localisée $l p\left(\mathscr{F}, \tau, \sigma ; \mathscr{H}, F_{i}\right)$ de $L P(\mathscr{F})$ est définie comme suite:

DÉFInITION 2.2 (Classe localisée de $L P(\mathscr{F})$ ).

$$
l p\left(\mathscr{F}, \tau, \sigma ; \mathscr{H}, F_{i}\right)=\left(1 / 4 \pi^{2}\right)\left[\left.f_{D^{2}}\left(\omega^{\prime} \wedge d \omega^{\prime}\right)\right|_{U_{i}}\right] \in H^{1}\left(F_{i} ; R\right)
$$

où $D^{2}$ est muni de l'orientation naturelle de $D^{2}(\subset C)$.

Et on obtient facilement:

LEMme 2.4. La classe localisée de $L P(\mathscr{F})$ ne dépend pas du choix de la connexion $\bar{\omega}^{\prime}$ sur $P$ satisfaisant les conditions du lemme 2.2.

THÉORÉme 2.5. Soit $\lambda$ un homomorphisme de sorte que le diagramme

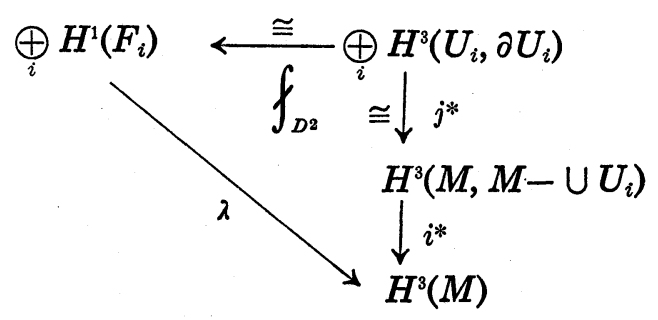

est commutatif, alors

$$
\lambda\left(\sum_{i} l p\left(\mathscr{F}, \tau, \sigma ; \mathscr{H}, F_{i}\right)\right)=L P(\mathscr{F})
$$

\section{§ 3. La classe localisée de $L P(\mathscr{F})$}

Dans ce paragraphe, nous considérons les interprétations géométriques de la classe localisée de $L P(\mathscr{F})$.

Soient $F$ une variété fermée et $D^{2}$ un disque de dimension 2 . Considérons un feuilletage riemannien $\mathscr{F}$ de codimension 2 sur $U=F \times D^{2}$ tel que $F(=F$ $\times o$ ) est une feuille compacte de $\mathscr{F}$. Sa structure transversalement riemanniene est désignée par $\tau$. Et nous supposons dans la suite que le fibré normal $\nu(\mathscr{F})$ est trivialisé par une section $\sigma=\left(\sigma_{1}, \sigma_{2}\right)$ du fibré $P$ de repères orthonormés de $\nu(\mathscr{F})$ et en plus que le feuilletage $\mathscr{F}$ admet une 1-extension singulière 
$(\mathscr{H}, \Sigma)$ dont l'ensemble de points singuliers $\Sigma$ est la feuille compacte $F$. Alors nous avons la classe localisée de la classe $L P(\mathscr{F})$;

$$
l p(\mathscr{F}, \tau, \sigma ; \mathscr{H}, F) \in H^{1}(F ; R) .
$$

Soit $X$ le champ de vecteurs sur un voisinage de la feuille compacte $F$ satisfaisant la condition (2) de la définition 2.1 pour la 1-extension singulière $(\mathscr{H}, \Sigma)$ donnée. Alors on peut supposer que $X$ est tangent à $x \times D^{2}$ en chaque point $(x, z) \in F \times D^{2}$. On a donc une famille $\left\{X_{x}\right\}_{x \in F}$ de champs de vecteurs sur $D^{2}$ qui s'annulent seulement en l'origine $o$ de $D^{2}$. Il est facile de voir que l'indice de $X_{x}$ ne dépend pas de $x(\in F)$. Désignons donc cette constante par $I(X)$ et appelons $t$-indice de $X$.

(I) En cas où $F=S^{1}$.

Tout d'abord, nous considérons le cas où $F=S^{1}$.

Pour chaque $s(\in R)$, désignons par $\mathscr{F}_{s}$ un feuilletage de codimension 2 sur $S^{1} \times D^{2}$ dont les feuilles sont les orbites d'un flot $\left\{\phi_{t}\right\}$ défini par

$$
\phi_{t}(x, z)=(x \cdot \exp (2 \pi t \sqrt{-1}), z \cdot \exp (2 \pi s t \sqrt{-1})),
$$

où $S^{1}=\partial D^{2}$ et $D^{2}=\{z \in C ;|z| \leqq 1\}$.

Alors, par le lemme 1.2, on a:

LEMME 3.1. Soit $\mathscr{F}$ le feuilletage riemannien précédent sur $U=F \times D^{2}$ $\left(=S^{1} \times D^{2}\right)$. Alors il existe un feuilletage $\mathscr{F}_{s}$ et un difféomorphisme feuilleté de classe $C^{\infty}$ d'un voisinage tubulaire saturé de $F$ sur un voisinage tubulaire saturé d'une feuille compacte $S^{1} \times$ o de $\mathscr{F}_{s}$, où o est l'origine de $D^{2}$.

Nous allons donc supposer que le feuilletage $\mathscr{F}_{s}$ est riemannien et satisfait les mêmes conditions que le feuilletage $\mathscr{F}$ satisfait.

Nous calculons $l p\left(\mathscr{F}_{s}, \tau, \sigma ; \mathscr{H}, F\right)\left(\left[S^{1}\right]\right) \in \boldsymbol{R}$.

Dans le cas où $F=S^{1}$, nous fixons l'orientation produite $O_{U}=O_{S^{1}} \times O_{D^{2}}$, où $O_{S^{1}}\left(\right.$ resp. $\left.O_{D^{2}}\right)$ est l'orientation naturelle de $S^{1}\left(=S O(2)=\partial D^{2}\right)\left(\right.$ resp. $\left.D^{2} \subset C\right)$.

Soient $s, s^{\prime}$ deux sections de $P$ définies sur $U-S^{1} \times o$ satisfaisant

$$
s(x, z)=s^{\prime}(x, z) \cdot F(x, z), \quad \operatorname{pour}(x, z) \in S^{1} \times D^{2},
$$

avec une fonction différentiable

$$
F: U-S^{1} \times o \longrightarrow S O(2) .
$$

Prenons un chemin fermé $c$ de $U-S^{1} \times o$ et désignons par $d\left(s, s^{\prime}\right)(c)$ le degré de l'application

$$
\left.F\right|_{c}: c\left(=S^{1}\right) \longrightarrow S O(2)\left(=S^{1}\right) .
$$

Nous l'appelons le nombre de rotation de $s$ à $s^{\prime}$ le long de c. On sait qu'il 
induit un homomorphisme

$$
d\left(s, s^{\prime}\right): \pi_{1}\left(U-S^{1} \times o\right) \longrightarrow Z,
$$

satisfaisant

$$
d\left(s, s^{\prime}\right)+d\left(s^{\prime}, s^{\prime \prime}\right)=d\left(s, s^{\prime \prime}\right) .
$$

Fixons les générateurs $e, e^{\prime}$ de $\pi_{1}\left(U-S^{1} \times o\right)$ représentés respectivement par les chemins fermés $c, c^{\prime}$;

$$
\begin{aligned}
c(t) & =(\exp (2 \pi t \sqrt{-1}), 1), \\
c^{\prime}(t) & =(1, \exp (2 \pi t \sqrt{-1})), \quad t \in[0,1]
\end{aligned}
$$

Nous avons:

THÉORÈme 3.2. Supposons que le repère mobile $\left(d \phi_{t} / d t, \sigma_{1}, \sigma_{2}\right)$ soit compatible avec l'orientation $O_{U}$ fixée. Et soit $\sigma_{o}=\left(\sigma_{o 1}, \sigma_{o 2}\right)$ une section de $P$ sur $U-S^{1} \times$ o telle que $\sigma_{o 1}=\partial / \partial r$ et $O_{D^{2}}$ est compatible avec $\sigma_{o}$, où $r=|z|, z \in D^{2}(\subset C)$. Alors,

(1) $\operatorname{lp}\left(\mathscr{F}_{s}, \tau, \sigma ; \mathscr{H}, F\right)\left(\left[S^{1}\right]\right)=\left(s-d\left(\sigma, \sigma_{o}\right)(e)\right) \cdot I(X)$;

(2) en particulier, si le nombre s est rationnel,

$$
l p\left(\mathscr{F}_{s}, \tau, \sigma ; \mathscr{H}, F\right)\left(\left[S^{1}\right]\right)=\left(-d\left(\sigma, \sigma_{o}\right)(L) / k\right) \cdot I(X) ;
$$

où $\left[S^{1}\right]$ est la classe de l'orientation $O_{S^{1}}$, L est une feuille compacte de $\mathscr{F}_{\text {s }}$ orientée par $d \phi_{t} / d t$ et différente de $F=S^{1} \times$ o et $k$ est l'ordre d'holonomie de $F$.

(II) En cas général.

D'abord, par le théorème 3.2, on a evidement:

THÉORÈme 3.3. Soit $D h: \pi_{1}(F) \rightarrow S O(2)$ l'holonomie linéaire de $F$. Alors

$$
l p(\mathscr{F}, \tau, \sigma ; \mathscr{H}, F)(a) \equiv \theta(a) \cdot I(X) \quad \bmod Z,
$$

où $a \in \pi_{1}(F)$ et $D h(a)=\exp (2 \pi \theta(a) \sqrt{-1})\left(\in S O(2)=S^{1} \subset C\right)$.

Nous disons qu'une section $s=\left(s_{1}, s_{2}\right)$ du fibré $P$ est transversalement projetable (par rapport à $(\mathscr{F}, \tau))$ sur un ensemble ouvert $O$ s'il existe une section $s_{\alpha}=\left(s_{\alpha 1}, s_{\alpha 2}\right)$ du fibré $P_{\alpha}$ pour chaque $\alpha$ satisfaisant

$$
\left(f_{\alpha}\right)_{*}\left(\left.s_{i}\right|_{U_{\alpha} \cap 0}\right)=s_{\alpha i}, \quad i=1,2 .
$$

Nous avons:

THÉORÈme 3.4. Supposons que le t-indice $I(X)$ du champ de vecteurs $X$ ne soit pas zéro et que la section $\sigma$ soit transversalement projetable (par rapport $\grave{a}(\mathscr{F}, \tau)$ ) sur un voisinage saturé $O d u$ bord $\partial U$ de $U$. Alors, la classe localisée 
$l p(\mathscr{F}, \tau, \sigma ; \mathscr{H}, F)$ est nulle dans $H^{1}(F ; R)$ si et seulement si il existe une section transversalement projetable $\sigma^{\prime}$ sur $U$ telle que $\sigma=\sigma^{\prime}$ sur $O$ et $\sigma^{\prime}$ est homotope $\grave{a}$ $\sigma$ par un homotopie dont le support est cotenue dans $U-\partial U$.

DÉMONSTRATION DES THÉORÈMES.

Soit $\bar{\omega}$ (resp. $\bar{\omega}^{\prime}$ ) la connexion sur le fibré $P$ définie par (1.2) (resp. la connexion sur $P$ satisfaisant le lemme 2.2 pour la 1-extension singulière ( $\mathscr{H}, \Sigma(=F))$ donnée).

D'abord, on remarque:

LeMme 3.5. Soit L une feuille du feuilletage $\mathscr{F}$.

(1) Les deux connexions $\left.\bar{\omega}\right|_{\left.P\right|_{L}}$ et $\left.\bar{\omega}^{\prime}\right|_{\left.P\right|_{L}}$ sur $\left.P\right|_{L}$ coincident et elles sont plates.

(2) Donc les deux revêtements horizontaux dans $(P, \bar{\omega})$ et $\left(P, \bar{\omega}^{\prime}\right) d^{\prime} u n$ chemin dans $L$, ils coincident, si les points initiaux coincident.

(3) En plus, l'holonomie $\pi_{1}(L) \rightarrow S O(2)$ de la connexion plate $\left.\bar{\omega}\right|_{P \mid L}\left(\right.$ et $\left.\left.\bar{\omega}^{\prime}\right|_{P \mid L}\right)$ est l'holonomie linéaire $D h: \pi_{1}(L) \rightarrow S O(2)$ de la feuille $L$ du feuilletage $\mathscr{F}$.

DÉmonstration. En effet, par la définition de $\bar{\omega}$, le sous-fibré $\left\{\bar{\theta}_{1}=\bar{\theta}_{2}=\right.$ $\bar{\omega}=0\}$ du fibré tangent $\tau\left(\left.P\right|_{L}\right)$ de $\left.P\right|_{L}$ est le fibré horizontal de $\left.\bar{\omega}\right|_{\left.P\right|_{L}}$ et complètement intégrable. Donc on a le point (1) par le lemme 2.2. D'où, on a les deux points (2) et (3).

c.q.f.d.

Supposons maintenant que $F=S^{1}$.

Nous considérons donc un feuilletage $\mathscr{F}_{s}$ du lemme 3.1.

Il existe alors une famille $\left\{c_{z}\right\}_{z \in D^{2}}$ de chemins dans les feuilles de $\mathscr{F}_{s}$ sur $S^{1} \times D^{2}$ déterminés par

$$
c_{z}(t)=\phi_{t}(1, z), \quad t \in[0,1],
$$

où $\phi_{t}$ est le flot sur $S^{1} \times D^{2}$ définisant $\mathscr{F}_{s}$ dans (3.1). Désignons par $\tilde{c}_{z}$ un unique revêtement horizontal de $c_{z}$ dans $\left(P, \bar{\omega}^{\prime}\right)$ dont le point initial est $\tilde{c}_{z}(0)$ $=\sigma\left(c_{z}(0)\right.$ ) (il est aussi horizontal pour $\bar{\omega}$ par le lemme 3.5). Alors il existe une fonction

$$
a:[0,1] \times D^{2} \longrightarrow R
$$

satisfaisant

$$
\begin{array}{ll}
a(0, z) \equiv 0 & \text { pour } z \in D^{2} ; \\
\text { et en posant } & A_{z}(t)=\exp (2 \pi a(t, z) \sqrt{-1})\left(\in S^{1}=S O(2)\right) \\
\tilde{c}_{z}(t)=\sigma\left(c_{z}(t)\right) \cdot A_{z}(t) . &
\end{array}
$$

Alors on a:

LEMME 3.6.

(1) $\left(\left(c_{z}\right) * \omega^{\prime}\right)(d / d t)=-2 \pi \partial a(t, z) / \partial t$; 
(2) En particulier, si s est un entier, on a

$$
(-1 / 2 \pi) \int_{c_{z}} \omega^{\prime}=a(1, z)=-d\left(\sigma, \sigma_{o}\right)\left(c_{z}\right)=s-d\left(\sigma, \sigma_{o}\right)(e) .
$$

DÉmonstration. D'abord, $\bar{\omega}^{\prime}\left(\left(\tilde{c}_{z}\right)_{*}(d / d t)\right)=0$, parce que $\tilde{c}_{z}$ est horizontal. Et par (3.5) on a

$$
\left.\frac{d}{d t} \sigma \circ c_{z}(t)\right|_{t=\tau}=\left(R_{A_{z}(\tau)-1}\right)_{*}\left(\left.\frac{d}{d t} \tilde{c}_{z}(t)\right|_{t=\tau}\right)+\left[\left.\frac{d}{d t}\left(A_{z}(\tau) \cdot A_{z}(t)^{-1}\right)\right|_{t=\tau}\right]_{\sigma \circ c_{z}(\tau)}^{*}
$$

et

$$
\left.\frac{d}{d t}\left(A_{z}(\tau) \cdot A_{z}(t)^{-1}\right)\right|_{t=\tau}=-2 \pi \partial a(t, z) /\left.\partial t\right|_{t=\tau}
$$

où $R_{g}$ (resp. $\left.Y^{*}\right)$ désigne la translation de $P$ à droit par $g(\in S O(2))$ (resp. le champ de vecteurs fondamental correspondant à un element $Y$ d'algèbre de Lie de $S O(2))$. Par suite, on a

$$
\left(\left(c_{z}\right) * \omega^{\prime}\right)(d / d t)=\bar{\omega}^{\prime}\left(\left(\sigma \circ c_{z}\right)_{*}(d / d t)\right)=-2 \pi \partial a(t, z) / \partial t .
$$

On a donc le point (1).

Démontrons le point (2).

Supposons que $s$ est un entier. Le feuilletage $\mathscr{F}_{s}$ est alors sans holonomie et les chemins $c_{z}$ sont fermés. Donc, par le lemme $3.5(3),\left\{\tilde{c}_{z}(t)\right\}$ définit une section $\tilde{\sigma}$ de $P$ sur $U=S^{1} \times D^{2}$. Par suite, si on compare (3.5) avec la définition du nombre de rotation $d(\tilde{\sigma}, \sigma)\left(c_{z}\right)$, on a

$$
a(1, z)=d(\tilde{\sigma}, \sigma)\left(c_{z}\right)(\in Z) ;
$$

en particulier, $a(1, z)$ est constante. Et par la formule (3.2), on a

$$
d(\tilde{\sigma}, \sigma)\left(c_{z}\right)=d\left(\tilde{\sigma}, \sigma_{o}\right)\left(c_{z}\right)+d\left(\sigma_{o}, \sigma\right)\left(c_{z}\right) .
$$

D'autre part, par la définition des sections $\tilde{\sigma}$ et $\sigma_{o}$, elles sont horizontaux le long de chemins $c_{z}(z \neq 0)$. Donc on a

$$
d\left(\tilde{\sigma}, \sigma_{o}\right)\left(c_{z}\right)=0, \quad \text { pour } z \neq o .
$$

Par conséquent,

$$
\begin{aligned}
a(1, z) & =d(\tilde{\sigma}, \sigma)\left(c_{z}\right)=d\left(\sigma_{o}, \sigma\right)\left(c_{z}\right) \\
& =d\left(\sigma_{o}, \sigma\right)(e)+s \cdot d\left(\sigma_{o}, \sigma\right)\left(e^{\prime}\right),
\end{aligned}
$$

parce que $\left[c_{z}\right]=e+s \cdot e^{\prime}$ dans $\pi_{1}\left(U-S^{1} \times o\right)$ pour $z \neq 0$. Et il est facile de voir que $d\left(\sigma_{o}, \sigma\right)\left(e^{\prime}\right)=1$, on a donc 


$$
a(1, z)=-d\left(\sigma, \sigma_{o}\right)\left(c_{z}\right)=s-d\left(\sigma, \sigma_{o}\right)(e) .
$$

Puisque $a(1, z)$ est constante, on a le point (2).

c.q.f.d.

DÉMONSTRATION DU THÉORÈME 3.2.

D'abord, par le théorème de Fubini ([6]), on a

$$
\begin{aligned}
l p\left(\mathscr{F}_{s}, \tau, \sigma ; \mathscr{H}, F\right)\left(\left[S^{1}\right]\right) & =\left(1 / 4 \pi^{2}\right)\left(f_{D^{2}} \omega^{\prime} \wedge d \omega^{\prime}\right)\left(\left[S^{1}\right]\right) \\
& =\left(1 / 4 \pi^{2}\right) \int_{\left(U, O_{U}\right)} \omega^{\prime} \wedge d \omega^{\prime}
\end{aligned}
$$

où $O_{U}$ est l'orientation produite $O_{S^{1}} \times O_{D^{2}}$ de $U=S^{1} \times D^{2}$.

Soit

$$
\phi:[0,1] \times D^{2} \longrightarrow U=S^{1} \times D^{2}
$$

une application définie par

$$
\phi(t, z)=\phi_{t}(1, z)=(\exp (2 \pi t \sqrt{-1}), z \cdot \exp (2 \pi s t \sqrt{-1})) .
$$

Alors, d'après le point (1) du lemme 3.6 , on a

$$
\phi^{*}\left(\omega^{\prime} \wedge d \omega^{\prime}\right)=-2 \pi \frac{\partial}{\partial t} a(t, z) d t \wedge \phi^{*}\left(d \omega^{\prime}\right),
$$

parce qu'on sait que $(-1 / 2 \pi) d \omega^{\prime}=e\left(\bar{\Omega}^{\prime}\right)$ et que la connexion $\bar{\omega}^{\prime}$ est transversalement projetable, la forme $e\left(\bar{\Omega}^{\prime}\right)$ est donc "transversalement projetable", d'où, $i(Z) e\left(\bar{\Omega}^{\prime}\right)=0$ pour tous $Z \in \tau\left(\mathscr{F}_{s}\right)$, où $e$ est le polynôme de Pfaff normalisé mentionné dans (1.4).

En plus, en appliquant un théorème de Stokes, on a

$$
(-1 / 2 \pi) \int_{\left(t \times D^{2}, o_{\left.D^{2}\right)}\right.} \phi^{*}\left(d \omega^{\prime}\right)=\int_{\left(x \times D^{2}, o_{\left.D^{2}\right)}\right.} e\left(\bar{\Omega}^{\prime}\right)=I\left(X_{x}\right)=I(X),
$$

pour tous $t(\in[0,1])$ et $x\left(\in S^{1}\right)$ fixes, où $I\left(X_{x}\right)$ est l'indice du champ de vecteurs $X_{x}$ en l'origine. En effet, si on considére une section $\sigma^{\prime}=\left(\sigma_{1}^{\prime}, \sigma_{2}^{\prime}\right)$ du fibré $P$ définie sur un voisinage du bord de $S^{1} \times D^{2}$ telle que $\sigma_{1}^{\prime}=f \cdot X$ pour une fonction $f$, on rappelle qu'elle est horizontale pour la connexion $\bar{\omega}^{\prime}$ et que $d\left(\sigma^{\prime}, \sigma\right)$ $\left(S_{x}^{1}\right)=I\left(X_{x}\right)$ pour tous $x\left(\in S^{1}\right)$, où $S_{x}^{1}=\partial\left(x \times D^{2}\right)$ est muni de l'orientation induitée de $O_{S^{1}} \times O_{D^{2}}$. On obtient alors (3.8) par la même manière qu'on a le point (2) du lemme 3.6.

(i ) En cas où s est un entier.

En général, on remarque d'abord que, dans (3.7), la seule fonction qui contient la variable $t$ est $\partial a(t, z) / \partial t$, parce que la forme $e\left(\bar{\Omega}^{\prime}\right)\left(=(-1 / 2 \pi) d \omega^{\prime}\right)$ est transversalement projetable. 
Alors, d'après le point (2) du lemme 3.6, on a par (3.6), (3.7) et (3.8):

$$
\begin{aligned}
l p\left(\mathscr{F}_{s}, \tau, \sigma ; \mathscr{H}, F\right)\left(\left[S^{1}\right]\right) & =\left(1 / 4 \pi^{2}\right) \int_{\left(U, o_{U}\right)} \omega^{\prime} \wedge d \omega^{\prime} \\
& =\left(1 / 4 \pi^{2}\right) \int_{[0,1] \times D^{2}} \phi^{*}\left(\omega^{\prime} \wedge d \omega^{\prime}\right) \\
& =(-1 / 2 \pi) \int_{D^{2}}\left\{\left(\int_{0}^{1} \frac{\partial}{\partial t} a(t, z) d t\right) \times \phi^{*}\left(d \omega^{\prime}\right)\right\} \\
& =\left(s-d\left(\sigma, \sigma_{o}\right)(e)\right)\left\{(-1 / 2 \pi) \int_{D^{2}} \phi^{*}\left(d \omega^{\prime}\right)\right\} \\
& =\left(s-d\left(\sigma, \sigma_{o}\right)(e)\right) \cdot I(X) \\
& \left(=-d\left(\sigma, \sigma_{o}\right)\left(c_{z}\right) \cdot I(X), \text { pour } z \neq o\right) .
\end{aligned}
$$

On a donc le théorème dans ce cas-là.

(ii) En cas où s est un nombre rationnel.

Posons $s=l / k$, où $k$ et $l$ sont des entiers avec $k>0$ et $(k, l)=1$.

Le feuilletage $\mathscr{F}_{s}$ se relève au feuilletage $\mathscr{F}_{l}$ sur un revêtement à $k$-feuillets $\hat{U}=\hat{S}^{1} \times D^{2}$ de $U=S^{1} \times D^{2}$. Désigons par $\hat{\tau}, \hat{\sigma}, \hat{\sigma}_{o}, \hat{\mathscr{H}}, \hat{F}, \hat{X}$ les relèvements naturels de $\tau, \sigma, \sigma_{o}, \mathscr{H}, F, X$ sur $\hat{U}$ respectivement.

On remarque que le relèvement $\mathscr{F}_{l}$ de $\mathscr{F}_{s}$ est au cas (i) précédent, alors on a

$$
\begin{aligned}
l p\left(\mathscr{F}_{l}, \hat{\tau}, \hat{\sigma} ; \hat{\mathscr{H}}, \hat{F}\right)\left(\left[\hat{S}^{1}\right]\right) & =\left(l-d\left(\hat{\sigma}, \hat{\sigma}_{o}\right)(\hat{e})\right) \cdot I(\hat{X}) \\
& =-d\left(\hat{\sigma}, \hat{\sigma}_{o}\right)\left(\hat{c}_{z}\right) \cdot I(\hat{X}) ;
\end{aligned}
$$

où $\hat{e}$ (resp. $\hat{c}_{z}$ ) est le relèvement de $e$ (resp. $c_{z}$ ) et [ $\left.\hat{S}^{1}\right]$ est la classe de l'orientation relevée de l'orientation $O_{S^{1}}$ donnée. En plus, on a clairement

$$
d\left(\hat{\sigma}, \hat{\sigma}_{o}\right)(\hat{e})=k \cdot d\left(\sigma, \sigma_{o}\right)(e), \quad I(\hat{X})=I(X) ;
$$

et

$$
l p\left(\mathscr{F}_{l}, \hat{\tau}, \hat{\sigma} ; \hat{\mathscr{H}}, \hat{F}\right)\left(\left[\hat{S}^{1}\right]\right)=k \cdot \operatorname{lp}\left(\mathscr{F}_{s}, \tau, \sigma ; \mathscr{H}, F\right)\left(\left[S^{1}\right]\right)
$$

où $\left[S^{1}\right]$ est la classe de l'orientation $O_{S 1}$.

Par conséquent, on a

$$
l p\left(\mathscr{F}_{s}, \tau, \sigma ; \mathscr{H}, F\right)\left(\left[S^{1}\right]\right)=\left(s-d\left(\sigma, \sigma_{o}\right)(e)\right) \cdot I(X) .
$$

Le point (2) est maintenant claire par (3.10) et (3.11).

On a aussi le théorème dans ce cas.

(iii) En cas où s est un nombre irrationel.

D'abord, considérons une famille différentiable $\left\{\mathscr{F}_{s+t}\right\}_{t}$ de feuilletages de classe $C^{\infty}$ sur $U=S^{1} \times D^{2}$. 
On peut supposer dans la suite que le fibré normal $\nu\left(\mathscr{F}_{s}\right)$ est aussi le fibré normal de $\mathscr{F}_{s+t}$ pour tous $t$ petits.

On remarque que l'application d'holonomie $f$ de la feuille compacte de $\mathscr{F}_{s}$ est un isométrie de $D^{2}$ muni d'une métrique riemannienne déterminée par la structure transversalement riemannienne $\tau$ de $\mathscr{F}_{s}$. Alors, l'ordre de l'isométrie $f$ est infini et le groupe d'isométries de $D^{2}$ contient donc $S O(2)$, parce que $s$ est irrationnel. Par suite, les feuilletages $\mathscr{F}_{s+t}$ admettent les structures transversalement riemanniennes $\tau_{s+t}$ telles que les métriques quasifibrées $g_{s+t}$ des fibrés normaux de $\left(\mathscr{F}_{s+t}, \tau_{s+t}\right)$ ne dépendent pas de $t$, où on a supposé que $\nu\left(\mathscr{F}_{s+t}\right)=\nu\left(\mathscr{F}_{s}\right)$.

Soit maintenant $X$ le champ de vecteurs qui détermine la 1-extension singulière $(\mathscr{H}, F)$ de $\mathscr{F}_{s}$. Puisque $s$ est irrationnel, le champ de vecteurs $X$ détermine encore des 1-extensions singulières $\left(\mathscr{H}_{s+t}, F\right)$ pour tous $\mathscr{F}_{s+t}$ telles que $\tau\left(\mathscr{H}_{s+t}\right)=\tau\left(\mathscr{F}_{s+t}\right) \oplus \boldsymbol{R} \cdot X$ sur $U-F$.

Par conséquent, on obtient une famille différentiable de connexions $\left\{\bar{\omega}_{s+t}^{\prime}\right\}$ sur le fibré principal $P$ des repères de $\nu\left(\mathscr{F}_{s}\right)\left(=\nu\left(\mathscr{F}_{s+t}\right)\right)$ orthonormés pour la métrique $g_{s}\left(=g_{s+t}\right)$ telle que les connexions $\bar{\omega}_{s+t}^{\prime}$ satisfont le lemme 2.2 pour $\left(\mathscr{H}_{s+t}, F\right)$. On a donc une fonction différentiable en $t$

$$
l p\left(\mathscr{F}_{s+t}, \tau_{s+t}, \sigma ; \mathscr{H}_{s+t}, F\right)\left(\left[S^{1}\right]\right)=\left(1 / 4 \pi^{2}\right) \int_{S 1 \times D^{2}}\left(\omega_{s+t}^{\prime}\right) \wedge d\left(\omega_{s+t}^{\prime}\right)
$$

où $\omega_{s+t}^{\prime}=\sigma^{*} \bar{\omega}_{s+t}^{\prime}$.

Le point (1) est maintenant claire par les considérations pour les cas (i) et (ii) précédents.

On a donc le théorème 3.2.

DÉMONSTRATION DU THÉORÈME 3.4.

Supposons d'abord

$$
l p(\mathscr{F}, \tau, \sigma ; \mathscr{H}, F)=0 \quad \text { dans } H^{1}(F ; \boldsymbol{R}) .
$$

Fixons un point $x_{0}$ de $F$. Soient $x$ un point de $F$ et $l$ un chemin simple tel que $l(0)=x_{o}$ et $l(1)=x$. Par la manière analogue au cas où $F=S^{1}$ pour le chemine $l$, on obtient une famille $\left\{l_{z}\right\}$ de chemins dans les feuilles et une fonction différentiable

$$
a_{l}:[0,1] \times D^{2} \longrightarrow \boldsymbol{R}
$$

satisfaisant

$$
a_{l}(0, z) \equiv 0 \quad \text { et } \quad \tilde{l}_{z}(t)=\sigma\left(l_{z}(t)\right) \cdot \exp \left(2 \pi a_{l}(t, z) \sqrt{-1}\right),
$$

pour les revêtements horizontaux $\left\{\tilde{l}_{z}\right\}$ de $\left\{l_{z}\right\}$. Puisque $I(X) \neq 0$, il est facile de voir que l'hypothèse $(*)$ implique, par le théorème 3.2 et le lemme 3.6 (2), 
qu'il existe une fonction différentiable

$$
\Phi: U\left(=F \times D^{2}\right) \longrightarrow R
$$

déterminée par

$$
\Phi(x, z)=a_{l}(1, z) \quad \operatorname{pour}(x, z) \in F \times D^{2} .
$$

Donc, si on prend une section $\sigma^{\prime}$ sur $U=F \times D^{2}$ définie par

$$
\sigma^{\prime}(x, z)=\sigma(x, z) \cdot \exp (2 \pi \Phi(x, z) \sqrt{-1}) \quad \operatorname{pour}(x, z) \in F \times D^{2},
$$

elle est transversalement projetable sur $U$.

On remarque que $\sigma \circ l_{z}$ est un revêtement horizontal de $l_{z}$ sur le voisinage saturé $O$ où la section $\sigma$ est transversalement projetable. Alors on a

$$
\Phi(x, z)=0 \quad \operatorname{pour}(x, z) \in O .
$$

Donc la section $\sigma^{\prime}$ satisfait

$$
\sigma^{\prime}=\sigma \quad \operatorname{sur} O .
$$

Et en plus $\sigma$ est homotope à $\sigma^{\prime}$ par l'homotopie $\sigma_{t}$ construit par

$$
\sigma_{t}(x, z)=\sigma(x, z) \cdot \exp (2 \pi t \Phi(x, z) \sqrt{-1}) .
$$

Inversement, soit $\sigma^{\prime}$ une section satisfaisant les conditions du théorème. Alors, par l'existence d'homotopie, on a d'abord

$$
l p(\mathscr{F}, \tau, \sigma ; \mathscr{H}, F)=l p\left(\mathscr{F}, \tau, \sigma^{\prime} ; \mathscr{H}, F\right) \quad \text { dans } H^{1}(F ; R) .
$$

En plus, puisque $\sigma^{\prime}$ est transversalement projetable et on a le théorème 3.2 et le lemme $3.6(2)$, on a

$$
l p\left(\mathscr{F}, \tau, \sigma^{\prime} ; \mathscr{H}, F\right)([c])=0,
$$

pour tous chemins simples fermés $c$ de $F$. Par conséquent,

$$
l p(\mathscr{F}, \tau, \sigma ; \mathscr{H}, F)=0 \quad \text { dans } H^{1}(F ; R) .
$$

On a donc le théorème 3.4 .

c.q.f.d.

\section{§4. Démonstration du théorème A}

Soit $\mathscr{F}$ un feuilletage riemannien de codimension 2 sur une variété orientable fermée $M$ de dimension 3 dont le fibré normal $\nu(\mathscr{F})$ est trivialisé par une section $\sigma=\left(\sigma_{1}, \sigma_{2}\right)$ du fibré de repères orthonormés de $\nu(\mathscr{F})$. La structure transversalement riemannienne de $\mathscr{F}$ est désignée par $\tau$. 
Nous supposons dans ce paragraphe que toutes les feuilles du feuilletage $\mathscr{F}$ sont compactes et démontrons le théorème $\mathrm{A}$.

Désignons par $n$ le nombre de feuilles ayant d'holonomie.

La variété $M$ est orientable de dimension 3 et le fibré normal $\nu(\mathscr{F})$ est trivial, les feuilles sont donc les orbites d'un flot. En plus, les orbites sont fermées par l'hypothèse, donc les feuilles sont les orbites d'une $S^{1}$-action $\Phi=$ $\left\{\phi_{\theta}\right\}_{\theta \in S_{1}}$ de $M([4])$. On peut supposer que l'action $\Phi$ est effective. Le nombre d'orbites exceptionnelles de $\Phi$ n'est que le nombre de feuilles ayant d'holonomie, c'est donc $n$. On sait qu'une telle $S^{1}$-action effective est déterminée par les invariants (orientés) suivants ([15], [16]):

$$
\left\{b ;(\varepsilon, g, f, t) ;\left(\alpha_{1}, \beta_{1}\right), \cdots,\left(\alpha_{n}, \beta_{n}\right)\right\} .
$$

Nous les rappelons dans la suite.

1. $(\varepsilon, g, f, t)$. Le nombre d'orbites exceptionnelles spéciaux (resp. de points fixes) est désigné par $t$ (resp. $f$ ) et $g$ (resp. $\varepsilon$ ) est le genre (resp. l'orientabilité) de l'espace d'orbites $M^{*}$. Puisque la variété $M$ est orientable et le fibré $\nu(\mathscr{F})$ est trivial, on a

$$
(\varepsilon, g, f, t)=(o, g, 0,0)
$$

c'est-à-dire que l'espace d'orbites $M^{*}$ est topologiquement une surface orientable fermée $T_{g}$ de genre $g$.

2. $\left(\alpha_{i}, \beta_{i}\right)$. Définissons une $S^{1}$-action (l'action linéaire standarde de type $(k, l))$ sur $S^{1} \times D^{2}$ par

$$
\exp (t \sqrt{-1}) \times(x, z)=(x \cdot \exp (k t \sqrt{-1}), z \cdot \exp (l t \sqrt{-1}))
$$

où $S^{1}=\partial D^{2}, D^{2}=\{z \in C ;|z| \leqq 1\}, 0<l<k$ et $k, l$ sont des nombres premiers entre eux.

Soit $O_{M}$ l'orientation de $M$ qui est compatible avec le repère mobile $\left(d \phi_{\theta} / d \theta, \sigma_{1}, \sigma_{2}\right)$.

Désignons par $F_{1}, \cdots, F_{n}$ les $n$-orbites exceptionnelles et par $U_{1}, \cdots, U_{n}$ des voisinages tubulaires équivariants de $F_{1}, \cdots, F_{n}$ respectivement. Alors, pour chaque $i(=1, \cdots, n)$, l'action $\left.\Phi\right|_{U_{i}}$ (resp. l'orientation $O_{U_{i}}$ induitée de $O_{M}$ ) sur $U_{i}$ s'identifie avec une unique action linéaire standarde de type $\left(k_{i}, l_{i}\right)$ (resp. l'orientation produite $O_{S^{1}} \times O_{D^{2}}$ ) sur $S^{1} \times D_{i}^{2}$ par un difféomorphisme de $U_{i} \operatorname{sur} S^{1} \times D_{i}^{2}$. Les couples $\left(\alpha_{1}, \beta_{1}\right), \cdots,\left(\alpha_{n}, \beta_{n}\right)$ satisfont par la définition

$$
\alpha_{i}=k_{i}, 0<\beta_{i}<\alpha_{i} \quad \text { et } \quad \beta_{i} \cdot l_{i} \equiv 1 \bmod \left(\alpha_{i}\right),
$$

pour $i=1, \cdots, n$; où $\beta_{i} \in Z$

3. Interprétation de $b(\in Z)$. Soient $F_{0}$ une orbite principale et $U_{0}$ un voisinage tubulaire équivariant de $F_{0}$. Et on identifie aussi l'action $\left.\Phi\right|_{U_{0}}$ 
(resp. l'orientation $O_{U_{0}}$ induitée de $O_{M}$ ) sur $U_{0}$ avec l'action linéaire standarde de type $(1,0)$ (resp. l'orientation produite $O_{S^{1}} \times O_{D^{2}}$ ) sur $S^{1} \times D_{0}^{2}$ par un difféomorphisme de $U_{0}$ sur $S^{1} \times D_{0}^{2}$. Soit

$$
p: M \longrightarrow M^{*}\left(\approx T_{g}\right)
$$

la projection. Posons

$$
D_{j}^{*}=p\left(D_{j}^{2}\right) \quad \text { pour } j=0,1, \cdots, n,
$$

où on suppose que $D_{j}^{2} \subset U_{j}$ par le difféomorphisme de $U_{j}$ sur $S^{1} \times D_{j}^{2}$. On peut supposer que les $(n+1)$-disques $D_{0}^{*}, \cdots, D_{n}^{*}$ sont disjoints. Alors, le fibré principal en $S O(2)$

$$
\left.p\right|_{M_{n}}: M_{n}\left(=M-\left(U_{0} \cup U_{1} \cup \cdots \cup U_{n}\right)\right) \longrightarrow M_{n}^{*}\left(=T_{g}-\left(D_{0}^{*} \cup D_{1}^{*} \cup \cdots \cup D_{n}^{*}\right)\right)
$$

admet une section

$$
\iota: M_{n}^{*} \longrightarrow M_{n}
$$

satisfaisant les relations homologiques suivantes:

$$
m_{0} \sim q_{0}+b \cdot h ; \quad m_{i} \sim \alpha_{i} \cdot q_{i}+\beta_{i} \cdot h, \quad i=1, \cdots, n ;
$$

où $m_{j}\left(\operatorname{resp} . q_{j}\right)(j=0,1, \cdots, n)$ est $\partial D_{j}^{2}$ orienté par l'orientation induitée de $O_{S^{1}} \times O_{D_{j}^{2}}\left(\right.$ resp. $\iota\left(\partial D_{j}^{*}\right)$ orienté opposément à l'orientation du bord de $M_{n}^{*}$ qui est induitée de celle de $M_{n}^{*}$ déterminée par $\left.\left(\sigma_{1}, \sigma_{2}\right)\right)$ et $h$ est l'orbite principale orientée par $\left(d \phi_{\theta} / d \theta\right)$.

On a montré:

LEMme 4.1. Les feuilles de $\mathscr{F}$ sont les orbites d'une $S^{1}$-action effective dont les invariants (orientés) sont

$$
\left\{b ;(o, g, 0,0) ;\left(\alpha_{1}, \beta_{1}\right), \cdots,\left(\alpha_{n}, \beta_{n}\right)\right\} ;
$$

et ils satisfont (4.2) et (4.3).

Supposons que le feuilletage $\mathscr{F}$ sur $M$ satisfait le lemme 4.1. Alors, on a les lemmes suivants. Les démonstrations seront données à la fin de ce paragraphe.

Lemme 4.2. Pour le premier nombre de Betti $b_{1}(M)$ de $M$, on $a$ :

(1) si $b+\sum_{i=1}^{n} \beta_{i} / \alpha_{i} \neq 0 ; b_{1}(M)=2 g$,

(2) si $b+\sum_{i=1}^{n} \beta_{i} / \alpha_{i}=0 ; b_{1}(M)=2 g+1$.

LEMme 4.3. Si $[M]$ est la classe de l'orientation $O_{M}$ qui est compatible avec le repère mobile $\left(d \phi_{\theta} / d \theta, \sigma_{1}, \sigma_{2}\right)$, on a 


$$
L P(\mathscr{F})([M])=d \cdot\left\{-2(1-g)+\sum_{i=1}^{n}\left(1-1 / \alpha_{i}\right)\right\}
$$

où $d \in \boldsymbol{Z}$.

LEMME 4.4. L'entier $d$ satisfait les équations suivantes:

(1) $\left(b+\sum_{i=1}^{n} \beta_{i} / \alpha_{i}\right) \cdot d=-2(1-g)+\sum_{i=1}^{n}\left(1-1 / \alpha_{i}\right)$;

(2) si $n>0 ; d / \alpha_{i} \equiv-l_{i} / k_{i} \bmod Z$ pour $i=1, \cdots, n$, où $\left(k_{i}, l_{i}\right)$ et $\left(\alpha_{i}, \beta_{i}\right)$ satisfont (4.2).

En supposant que les lemmes soient vrais, nous démontrons le théorème $\mathrm{A}$.

DÉmonstration DU THÉORÈme A.

(1) En cas où $n=0$. Il est claire que le feuilletage $\mathscr{F}$ est 1-extendable si $g=1$. Et, si $\mathscr{F}$ est 1 -extendable, la classe $L P(\mathscr{F})$ est nulle par le lemme 1.1. Supposons donc la classe $L P(\mathscr{F})$ est nulle. Alors, par les lemmes 4.3 et 4.4 , on a $g=1$. Donc les trois conditions (i), (ii) et (iii) sont équivalentes.

Par l'interprétation de $b$, si $b=0$, alors $g=1$. Donc, $b_{1}(M)$ n'est pas 1 par le lemme 4.2.

(2) En cas où $n>0$. Si $g \geqq 1$, alors on a

$$
-2(1-g)+\sum_{i=1}^{n}\left(1-1 / \alpha_{i}\right) \geqq n / 2>0 \text {. }
$$

Par les lemmes 4.2 et $4.4, b_{1}(M)$ est donc $2 g$ si $g \geqq 1$, et $b_{1}(M)=0$ ou 1 si $g=0$. Par conséquent, $b_{1}(M)=2 g$ ou 1 .

On remarque que $d \neq 0$, par le point (2) du lemme 4.4. Alors, par les lemmes $4.2,4.3$ et (4.4), on a

$$
\begin{aligned}
& \text { (i) si } b_{1}(M)=2 g, L P(\mathscr{F})([M]) \neq 0 \text { et } \\
& L P(\mathscr{F})([M])=\left\{-2(1-g)+\sum_{i=1}^{n}\left(1-1 / \alpha_{i}\right)\right\}^{2} /\left(b+\sum_{i=1}^{n} \beta_{i} / \alpha_{i}\right),
\end{aligned}
$$

(ii) si $b_{1}(M)=1, L P(\mathscr{F})([M])=0$.

Par conséquent, on a le théorème $\mathrm{A}$.

c.q.f.d.

Remarque. Dans la condition $n>0$, la classe $L P(\mathscr{F})$ est nulle si et seulement si le feuilletage $\mathscr{F}$ est déterminé par l'une des trois $S^{1}$-actions avec les invariants suivants:

$$
\begin{aligned}
& g=0, b=-2(\text { resp. }-1,-2), n=4(\operatorname{resp} .3,3) \\
& \alpha_{i}=2(\text { resp. } 3,3), \beta_{i}=1(\text { resp. } 1,2), \text { pour tout } i .
\end{aligned}
$$

Nous démontrons maintenant les lemmes. 
DÉMONSTRATION DU LEMME 4.2.

On sait que le groupe fondamental de $M$ est un groupe à $(2 g+n+1)$ générateurs $a_{1}, b_{1}, \cdots, a_{g}, b_{g}, c_{1}, \cdots, q_{n}, h$ avec les relations suivantes ([16]):

$$
\begin{aligned}
& {\left[a_{j}, h\right]=\left[b_{j}, h\right]=\left[q_{i}, h\right]=1,} \\
& \left(q_{i}\right)^{\alpha_{i}} \cdot(h)^{\beta_{i}}=1, \quad \text { pour tout } j=1, \cdots, g, i=1, \cdots, n ;
\end{aligned}
$$

et

$$
q_{1} \cdots q_{n} \cdot\left[a_{1}, b_{1}\right] \cdots\left[a_{g}, b_{g}\right]=h^{b} .
$$

D'où, on a le lemme 4.2.

c.q.f.d.

DÉMONSTRATION DU LEMME 4.3.

Prenons un champ de vecteurs $X$ sur l'espace d'orbites $M^{*}\left(\approx T_{g}\right)$ satisfaisant les conditions suivantes:

(4.6) (1) les points singuliers de $X$ est $(n+1)$-points $x_{0}, x_{1}, \cdots, x_{n}$, où $x_{j}=$ $p\left(F_{j}\right)$ pour $j=0,1, \cdots, n$;

(2) les $n$-points $x_{1}, \cdots, x_{n}$ sont des cols pour $X$ (donc, si $I_{0}$ est l'indice de $X$ en $\left.x_{0}, n+I_{0}=2(1-g)\right)$;

(3) il existe un champ de vecteurs $X$ sur $M$ satisfaisant

$$
p_{*}\left(\left.X\right|_{M-\left(F_{1} \cup \cdots \cup F_{n}\right)}\right)=\left.X\right|_{M^{*}-\left\{x_{1}, \cdots, x_{n}\right)} .
$$

Alors, le champ de vecteurs $X$ défine une 1-extension singulière $(\mathscr{H}, \Sigma)$ satisfaisant les conditions suivantes:

(1) $\Sigma=F_{0} \cup F_{1} \cup \cdots \cup F_{n}$;

(2) le $t$-indice $I(X)$ de $X$ est 1 (resp. $\left.I_{0}\right)$ en $F_{i}(i=1, \cdots, n)$ (resp. $F_{0}$ ).

Par suite, par le théorème 3.2 , on $\mathrm{a}$

$$
\text { (1) } \begin{aligned}
l p\left(\mathscr{F}, \tau, \sigma ; \mathscr{H}, F_{i}\right)\left(\left[S^{1}\right]\right) & =\left(-d\left(\sigma, \sigma_{o}^{i}\right)\left(L^{i}\right) / k_{i}\right) \cdot 1 \\
& =l_{i} / k_{i}-d\left(\sigma, \sigma_{o}^{i}\right)\left(e_{i}\right), \quad \text { pour } i=1, \cdots, n ; \\
\text { (2) } \operatorname{lp}\left(\mathscr{F}, \tau, \sigma ; \mathscr{H}, F_{0}\right)\left(\left[S^{1}\right]\right) & =\left(-d\left(\sigma, \sigma_{o}^{0}\right)\left(L^{0}\right)\right) \cdot I_{0} ;
\end{aligned}
$$

où $\sigma_{o}^{0}, \sigma_{o}^{1}, \cdots, \sigma_{o}^{n}\left(\operatorname{resp} . L^{0}, L^{1}, \cdots, L^{n}\right.$ ) sont les sections du fibré $P$ (resp. les feuilles compactes orientées) mentionnées dans le théorème 3.2 respectivement et $e_{i}$ est le générateur de $\pi_{1}\left(U_{i}-S^{1} \times o\right)$ correspondant à $e$ défini par (3.3) pour $i=1, \cdots, n$.

En plus, $d\left(\sigma, \sigma_{0}^{j}\right)\left(L^{j}\right)$ ne dépend pas de $j . \quad$ En effet, si $\sigma^{\prime}=\left(\sigma_{1}^{\prime}, \sigma_{2}^{\prime}\right)$ est une section du fibré $P$ définie sur $M-\Sigma$ telle que $\sigma_{1}^{\prime}=f \cdot X$ pour une fonction différentiable $f, d\left(\sigma, \sigma^{\prime}\right)(L)$ ne dépend pas de feuille compacte sans holonomie $L$ 
qui est orientée par $\left(d \phi_{\theta} / d \theta\right)$ et en plus

$$
d\left(\sigma, \sigma_{o}^{j}\right)\left(L^{j}\right)=d\left(\sigma, \sigma^{\prime}\right)\left(L^{j}\right) \quad \text { pour } j=0,1, \cdots, n,
$$

parce que les deux sections $\sigma_{o}^{j}$ et $\sigma^{\prime}$ sont transversalement projetables.

Posons donc

$$
d=d\left(\sigma, \sigma_{o}^{j}\right)\left(L^{j}\right)\left(=d\left(\sigma, \sigma^{\prime}\right)\left(L^{j}\right)\right), \quad j=0,1, \cdots, n .
$$

Alors, par (4.8), on a

$$
\begin{aligned}
L P(\mathscr{F})([M]) & =d \cdot\left(-I_{0}-\sum_{i=1}^{n} 1 / k_{i}\right) \\
& =d \cdot\left(-2(1-g)+\sum_{i=1}^{n}\left(1-1 / k_{i}\right)\right),
\end{aligned}
$$

où $[M]$ est la classe de l'orientation $O_{M}$.

D'où, le résultat.

c.q.f.d.

DÉMONSTRATION DU LEMME 4.4.

D'abord, par (4.8) (1) et (4.9), on a

$$
-d / \alpha_{i} \equiv l_{i} / k_{i} \quad \bmod Z \quad \text { pour } i=1, \cdots, n \text {. }
$$

Démontrons donc le point (1) du lemme. Soient $\iota: M_{n}^{*} \rightarrow M_{n}$ la section satisfaisant (4.3) et $\sigma^{\prime}=\left(\sigma_{1}^{\prime}, \sigma_{2}^{\prime}\right)$ une section du fibré $P$ mentionnée dans la démonstration du lemme 4.3 qui satisfait $\sigma(a)=\sigma^{\prime}(a) \cdot A$ en un point $a \in M_{n}$ pour $A \in S O(2)$. Alors, il existe une fonction différentiable

$$
F: M_{n}^{*} \longrightarrow S O(2)
$$

déterminée par l'équation

$$
\sigma(\iota(x))=\sigma^{\prime}(\iota(x)) \cdot F(x) .
$$

Donc, le degré (de Brouwer) $d\left(\left.F\right|_{\partial M_{n}^{*}}, y\right)$ de $\left.F\right|_{\partial M_{n}^{*}}$ pour toute valeur régulière $y$ est zéro ([13]), c'est-à-dire,

$$
\sum_{j=0}^{n} d\left(\sigma, \sigma^{\prime}\right)\left(q_{j}\right)=0
$$

Posons

$$
d_{j}=d\left(\sigma, \sigma^{\prime}\right)\left(q_{j}\right), \quad j=0,1, \cdots, n \text {. }
$$

Alors, (4.11) s'écrit

$$
d_{0}+d_{1}+\cdots+d_{n}=0
$$


D'autre part, l'entier $d$ satisfait

$$
d=d\left(\sigma, \sigma^{\prime}\right)(h)
$$

par la définition (4.9), on a donc les équations suivantes par (4.3):

$$
\left\{\begin{array}{l}
-1=\alpha_{i} \cdot d_{i}+\beta_{i} \cdot d, \quad i=1, \cdots, n ; \\
-I_{0}=d_{0}+b \cdot d .
\end{array}\right.
$$

Par suite, on a

$$
\left(b+\sum_{i=1}^{n} \beta_{i} / \alpha_{i}\right) \cdot d+I_{0}+\sum_{i=1}^{n} 1 / \alpha_{i}=0
$$

D'où, le résultat.

c.q.f.d.

\section{§5. Dèmonstration du thèoréme $B$}

Soit $\mathscr{F}$ un feuilletage riemannien de codimension 2 sur une variété orientable fermée $M$ de dimension 3 muni d'une trivialisation $\sigma=\left(\sigma_{1}, \sigma_{2}\right) d u$ fibré $\nu(\mathscr{F})$ qui est orthonormée pour la métrique quasi-fibrée $g$ déterminée par la structure transversalement riemannienne $\tau$.

Nous supposons dans ce paragraphe que le feuilletage $\mathscr{F}$ est au cas (B) du théorème de Molino, et démontrons le théorème $B$.

D'abord, on remarque la trivialité de $\nu(\mathscr{F})$, alors on a:

LEMME 5.1. Pour ce feuilletage $\mathscr{F}$, on $a$ :

(1) les adhérences des feuilles de $\mathscr{F}$ détermine une 1-extension singulière $(\mathscr{H}, \Sigma)$;

(2) $\mathscr{F}$ possède seulement deux feuilles compactes $F_{1}, F_{2}$ donc $\Sigma=F_{1} \cup F_{2}$;

(3) les t-indices des champs de vecteurs en $F_{1}$ et $F_{2}$ définissant la 1extension singulière $(\mathscr{H}, \Sigma)$ (cf. Définition $2.1(2))$ sont égaux 1.

Fixons maintenant une orientation $O_{M}$ de $M$. Alors, il existe un flot $\left\{\phi_{t}\right\}$ dont les orbites sont les feuilles de $\mathscr{F}$, et on peut supposer que l'orientation $O_{M}$ est compatible avec le repère mobile $\left(d \phi_{t} / d t, \sigma_{1}, \sigma_{2}\right)$.

Par le lemme 5.1, on a:

LEMme 5.2. La variété orientée $M$ admet une décomposition feuilletée qui est compatible avec les orientations:

$$
(M, \mathscr{F})=\left(S_{1}^{1} \times D^{2}, \mathscr{F}_{s_{1}}\right) \bigcup_{f}\left(S_{2}^{1} \times D^{2}, \mathscr{F}_{s_{2}}\right),
$$

où $S_{i}^{1} \times D^{2}$ est muni d'un feuilletage $\mathscr{F}_{s_{i}}$ avec $s_{i}$ irrationnel et de l'orientation produite naturelle mentionnée dans le paragraphe 3 , et $f$ est un difféomorphisme recollant les tores de bords. 
Par la trivialité de $\nu(\mathscr{F})$ et le lemme 5.2, on a:

LEMME 5.3. Le groupe fondamentale de la variété M est un groupe cyclique d'ordre fini.

Et, en appliquant une interprétation de la classe localisée de $L P(\mathscr{F})$ du théorème $3.2 \mathrm{au}$ lemme 5.2, on $\mathrm{a}$ :

LEMME 5.4. Si la variété $M$ est simplement connexe, la classe de LazarovPasternack LP(F) du feuilletage $\mathscr{F}$ est non-nulle.

Il est maintenant claire que le théorème B est une conséquence immédiate des lemmes 5.3 et 5.4 .

Nous démontrons les lemmes.

DÉMONSTRATION DU LEMME 5.1.

D'abord, par la trivialité de $\nu(\mathscr{F})$, le feuilletage $\mathscr{F}$ est transversalement orientable.

En plus, d'après la classification de Molino (voir [14] p.305), on a:

(5.1) Si le feuilletage $\mathscr{F}$ est transversalement orientable (et si $\mathscr{F}$ est au cas (B)), il y a deux feuilles compactes. On a une application $\pi: M \rightarrow[0,1]$, où $\pi^{-1}(t)$ est l'adhérence d'une feuille, les feuilles compactes correspondant à $t=0$ ou 1 .

On a donc les points (1) et (2). Le point (3) est claire par le point (1). c.q.f.d.

DÉMONSTRATION DU LEMME 5.2.

Posons

$$
\tau(\mathscr{H})=\tau(\mathscr{F}) \oplus \nu \quad \text { sur } M-\Sigma .
$$

Le fibré normal $\nu(\mathscr{F})$ admet alors une décomposition orthogonale pour la métrique quasi-fibrée $g$

$$
\nu(\mathscr{F})=\nu \oplus \nu^{\prime} \quad \operatorname{sur} M-\Sigma .
$$

Alors, il est facile de voir qu'il existe une autre 1-extension singulière $\left(\mathscr{H}^{\prime}, \Sigma^{\prime}\right)$ satisfaisant

$$
\Sigma^{\prime}=\Sigma \quad \text { et } \quad \tau\left(\mathscr{H}^{\prime}\right)=\tau(\mathscr{F}) \oplus \nu^{\prime} \quad \text { sur } M-\Sigma \text {. }
$$

Donc, par le lemme 3.1 et (5.1), on a une décomposition feuilletée

$$
(M, \mathscr{F})=\left(S_{1}^{1} \times D^{2}, \mathscr{F}_{s_{1}}\right) \cup\left(T^{2} \times[0,1], \mathscr{F}^{\prime}\right) \cup\left(S_{2}^{1} \times D^{2}, \mathscr{F}_{s_{2}}\right),
$$

où $\mathscr{F}^{\prime}$ est un feuilletage produit $\mathscr{F}_{s}^{\prime} \times\{1$-points $\}$, et $\mathscr{F}_{s}^{\prime}$ est un feuilletage sur 
le tore $T^{2}$ déterminé par un folt sur le tore satisfaisant (3.1).

D'où, on a le résultat facilement.

c.q.f.d.

DÉMONSTRATION DU LEMME 5.3.

Soient $e_{1}, e_{1}^{\prime}$ (resp. $\left.e_{2}, e_{2}^{\prime}\right)$ les générateurs de $\pi_{1}\left(S_{1}^{1} \times D^{2}-S_{1}^{1} \times o\right.$ ) (resp. $\left.\pi_{1}\left(S_{2}^{1} \times D^{2}-S_{2}^{1} \times 0\right)\right)$ correspondant aux générateurs $e, e^{\prime}$ déterminés dans (3.3) respectivement. Alors, l'application

$$
f_{*}: \pi_{1}\left(\partial\left(S_{1}^{1} \times D^{2}\right)\right) \longrightarrow \pi_{1}\left(\partial\left(S_{2}^{1} \times D^{2}\right)\right)
$$

induitée du difféomorphisme $f$ satisfait

$$
\left(f_{*} e_{1}, f_{*} e_{1}^{\prime}\right)=\left(e_{2}, e_{2}^{\prime}\right)\left(\begin{array}{ll}
a & c \\
b & d
\end{array}\right)
$$

Maintenant, puisque le fibré normal $\nu(\mathscr{F})$ est trivial, il n'existe pas de sphère $S^{2}$ plongée transversalement au feuilletage $\mathscr{F}$. Il est donc claire que $c \neq 0$. Et, par le théorème de Van-Kampen, on a

$$
\pi_{1}(M)=Z_{|c|}, \quad c \neq 0 .
$$

DÉMONSTRATION DU LEMME 5.4.

D'abord, par l'hypothèse et (5.3), on a

$$
c= \pm 1
$$

Et, par l'orientabilité de $M$, on a

$$
\operatorname{det} f_{*}=a \cdot d-b \cdot c=-1 .
$$

D'autre part, par le théorème 3.2 et le lemme 5.2, on a:

(5.6) (1) $\quad l p\left(\mathscr{F}, \tau, \sigma ; \mathscr{H}, F_{i}\right)\left(\left[S_{i}^{1}\right]\right)=s_{i}-d_{i}$, pour $i=1,2$;

(2) $L P(\mathscr{F})([M])=\left(s_{1}-d_{1}\right)+\left(s_{2}-d_{2}\right)$;

où $d_{i}=d\left(\sigma, \sigma_{o}^{i}\right)\left(e_{i}\right) \in Z(i=1,2)$ et $[M]$ est la classe de l'orientation $O_{M}$.

En plus, par la définition des nombles de rotation $d_{1}, d_{2}$, ils satisfont les équations suivantes:

$$
d_{1}=a \cdot d_{2}-b ; \quad-1=c \cdot d_{2}-d
$$

On a donc, par (5.4), (5.5) et (5.7),

$$
c\left(d_{1}+d_{2}\right)=d-a-2, \quad \text { où } c= \pm 1 .
$$

Puis, on remarque que les feuilles du feuilletage de codimension 1 $\left.\mathscr{F}_{s_{1}}\right|_{\partial\left(S_{1}^{1} \times D^{2}\right)}$ (resp. $\left.\mathscr{F}_{s_{2}}\right|_{\partial\left(S_{2}^{1} \times D^{2}\right)}$ ) sur le tore du bord sont homologiquement $\left[e_{1}\right]+s_{1}\left[e_{1}^{\prime}\right]$ (resp. $\left[e_{2}\right]+s_{2}\left[e_{2}^{\prime}\right]$ ) et que le difféomorphisme $f$ conserve les feuille- 
tages. Alors, l'application induitée de $f$ en homoloqie avec coefficient réel conserve les "inclinaisons" $s_{1}$ et $s_{2}$, à savoir;

$$
s_{2}=\left(b+d \cdot s_{1}\right) /\left(a+c \cdot s_{1}\right) \text {. }
$$

Maintenant, si on suppose que $L P([M])=\left(s_{1}-d_{1}\right)+\left(s_{2}-d_{2}\right)$ soit zéro, $s_{1}$ est une solution irrationnelle d'équation suivante par (5.9):

$$
c x^{2}+\left(a+d-c\left(d_{1}+d_{2}\right)\right) x+\left(b-a\left(d_{1}+d_{2}\right)\right)=0 .
$$

Mais, par (5.4), (5.5) et (5.8), on a

$$
c x^{2}+\left(a+d-c\left(d_{1}+d_{2}\right)\right) x+\left(b-a\left(d_{1}+d_{2}\right)\right)=c\{x+c(a+1)\}^{2} .
$$

Ce qui est contradictoire.

c.q.f.d.

\section{§6. Dèmonstration du thèoréme $D$}

Soit $\mathscr{F}$ un feuilletage riemannien de codimension 2 sur une variété fermée $M$ de dimension 3 dont le fibré normal $\nu(\mathscr{F})$ est trivial.

Supposons dans ce paragraphe que toutes les feuilles de $\mathscr{F}$ soient partout denses dans $M$ et démontrons que le feuilletage $\mathscr{F}$ admet une 1-extension.

D'abord, on rappelle les équations (1.6)

$$
\left(\begin{array}{l}
d \theta_{1} \\
d \theta_{2}
\end{array}\right)=\left(\begin{array}{rr}
0 & \omega \\
-\omega & 0
\end{array}\right)\left(\begin{array}{l}
\theta_{1} \\
\theta_{2}
\end{array}\right) \text { et } \quad d \omega=f \theta_{2} \wedge \theta_{1}
$$

et que le feuilletage $\mathscr{F}$ est défini par

$$
\tau(\mathscr{F})=\left\{\theta_{1}=\theta_{2}=0\right\}
$$

On a facilement

$$
d f=f_{1} \theta_{1}+f_{2} \theta_{2}
$$

où $f_{1}$ et $f_{2}$ sont des fonctions différentiables. Alors, la fonction $f$ est une constante $C$, parce que les feuilles de $\mathscr{F}$ sont partout denses dans $M$. En résumé, on a

$$
\begin{aligned}
& \text { (1) } \tau(\mathscr{F})=\left\{\theta_{1}=\theta_{2}=0\right\} ; \\
& \text { (2) }\left(\begin{array}{l}
d \theta_{1} \\
d \theta_{2}
\end{array}\right)=\left(\begin{array}{rr}
0 & \omega \\
-\omega & 0
\end{array}\right)\left(\begin{array}{l}
\theta_{1} \\
\theta_{2}
\end{array}\right) \text { et } d \omega=C \theta_{2} \wedge \theta_{1} ; C \in \boldsymbol{R} .
\end{aligned}
$$

Dans ces conditions, le feuilletage $\mathscr{F}$ est appelé, suivant $R$. Blumenthal ([2]), transversalement euclidien (resp. elliptique, hyperbolique), si $C=0$ (resp. $C>0, C<0)$. 
Nous rappelons maintenant le théorème fondamental suivant pour tels feuilletages.

THÉORÈME (R. Blumenthal [2]). Soit $\mathscr{F}$ un feuilletage transversalement euclidien (resp. elliptique, hyperbolique) sur une variété fermée $M$. Soit $G=$ $S O(2) \dot{\times} R^{2}$ (resp. $\left.S O(3), S L(2 ; R) /\{ \pm 1\}\right)$ la composant connexe unitée du groupe des isométries de $B=G / S O(2)=R^{2}$ un plane euclidien (resp. $S^{2}$ une sphère naturelle, $H^{2}$ un demi-plane hyperbolique). Alors, le feuilletage $\tilde{\mathscr{F}}$ relevé de $\mathscr{F}$ sur le revêtement universel $\pi: \tilde{M} \rightarrow M$ est défini par une fibration localement triviale $q: \tilde{M} \rightarrow B$ à fibres connexes. De plus, il existe une représentation $\rho: \pi_{1}(M) \rightarrow G$ caractérisée par la relation suivante

$$
q(\alpha(\tilde{x}))=\rho(\alpha) \cdot q(\tilde{x}), \quad \text { pour } \tilde{x} \in \tilde{M},
$$

où $\alpha \in \pi_{1}(M)$ (resp. $\left.\rho(\alpha) \in G\right)$ s'identifie avec l'automorphisme de $\tilde{M}$ (resp. l'action isométrique de $G$ à gauche sur $B$ [11])).

On déduit de ce théorème:

ConollaIRE. Soient $F$ une feuille d'un tel feuilletage $\mathscr{F}$, et $\tilde{F}$ une feuille de $\tilde{\mathscr{F}}$ dans $\pi^{-1}(F)$. Soit $\Gamma$ l'image de $\rho$. Alors,

(1) la feuille $F$ est partout dense, si et seulement si l'orbite de $q(\tilde{F})(\in B)$ par $\Gamma$ est partout dense;

(2) le groupe d'holonomie de $F$ est isomorphe au sous-groupe d'isotropie de $\Gamma$ au point $q(\tilde{F})$.

Dans le théorème de Blumentbal, si $B=S^{2}$, la fibration $q: \tilde{M} \rightarrow B=S^{2}$ n'admet pas de section globale, parce que le fibré normal $\nu(\mathscr{F})$ est trivial. Donc, le groupe fondamental de la fibre n'est pas trivial, ceux des feuilles de $\tilde{\mathscr{F}}$ non plus. D'où, par (6.1), on a:

LEMme 6.1. Si le feuilletage riemannien $\mathscr{F}$ sur une variété fermée $M$ de dimension 3 dont les feuilles sont partout denses dans $M$, il est transversalement euclidien ou hyperbolique.

En plus, on a:

Lemme 6.2. Supposons qu'un feuilletage $\mathscr{F}$ soit transversalement euclidien ou hyperbolique sur une variété fermée $M$ de dimension 3 dont toutes les feuilles soient partout denses dans $M$. Alors, le feuilletage $\mathscr{F}$ admet une 1-extension.

DÉmonstration. Si le feuilletage $\mathscr{F}$ est transversalement hyperbolique, on a démontré dans [17] que l'adhérence $\bar{\Gamma}$ de $\Gamma$ dans $S L(2 ; R) /\{ \pm 1\}$ est conjuqué à un sous-groupe du groupe affine $A$ 


$$
A=\left\{\left(\begin{array}{cc}
a & b \\
0 & a^{-1}
\end{array}\right) ; a \neq 0, b \in R\right\} /\{ \pm 1\}
$$

et que le groupe $A$ conserve un feuilletage de codimension 1 sur $H^{2}$ dont les feuilles sont les droites horizontales. D'où, le résultat.

Supposons maintenant que $\mathscr{F}$ soit transversalement euclidien. Le feuilletage $\mathscr{F}$ est sans holonomie, parce que la dimension des feuilles est maintenant 1. Donc, d'après le point (2) du corollaire, aucun élément de $\Gamma$, en dehors de l'unité, n'a de point fixe dans le plan euclidien $B=\boldsymbol{R}^{2}$. De plus, on sait que l'action isométrique de $G=S O(2) \dot{\times} \boldsymbol{R}^{2}$ sur $R^{2}$ est donnée par l'équation

$$
g \cdot\left(\begin{array}{l}
x_{1} \\
x_{2}
\end{array}\right)=A \cdot\left(\begin{array}{l}
x_{1} \\
x_{2}
\end{array}\right)+\left(\begin{array}{l}
a_{1} \\
a_{2}
\end{array}\right)
$$

où

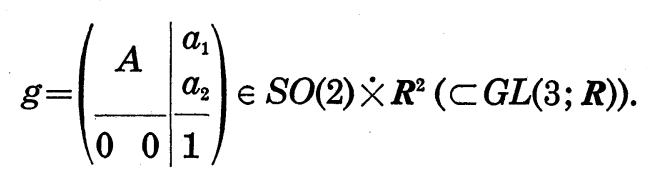

Par conséquent, le groupe $\Gamma$ n'est qu'un sous-groupe du groupe des translations parallèles. Et clairement il existe un feuilletage de codimension 1 sur $\boldsymbol{R}^{2}$ qui est conservé par toutes les translations parallèles. Par suite, on a une 1-extension de $\mathscr{F}$ en appliquant le théorème de Blumenthal. c.q.f.d.

Il est claire que le théorème $D$ est une conséquence immédiate des lemmes 6.1 et 6.2.

\section{§ 7. Exemple de Lazarov et Pasternack}

L'exemple suivant est donné dans [12].

D'abord, on identifie $S^{3}$ avec le groupe de Lie $S U(2)$.

Pour chaque $u$ avec $-1<u<1$, on a un feuilletage $\mathscr{F}_{u}$ sur $S^{3}$ dont les feuilles sont les orbites d'un flot $\phi_{t}^{u}$ défini par

$$
\phi_{t}^{u}(g)=\left(\begin{array}{cc}
\exp (u t \sqrt{-1}) & 0 \\
0 & \exp (-u t \sqrt{-1})
\end{array}\right) \cdot g \cdot\left(\begin{array}{cc}
\exp (t \sqrt{-1}) & 0 \\
0 & \exp (t-\sqrt{-1})
\end{array}\right)
$$

pour $t \in \boldsymbol{R}$ et $g \in S U(2)$. On sait que le feuilletage $\mathscr{F}_{u}$ est riemannien et son fibré normal $i\left(\mathscr{F}_{u}\right)$ est trivial.

Soient $X, \sigma_{1}, \sigma_{2}$ les champs de vecteurs invariants à gauche de $S U(2)$ correspondant aux éléments de l'algèbre de Lie

$$
\left(\begin{array}{cc}
\sqrt{-1} & 0 \\
0 & \sqrt{-1}
\end{array}\right),\left(\begin{array}{cc}
0 & -1 \\
1 & 0
\end{array}\right),\left(\begin{array}{cc}
0 & \sqrt{-1} \\
\sqrt{-1} & 0
\end{array}\right)
$$


respectivement et soit $\left[S^{3}\right]$ la classe de l'orientation de $S^{3}$ déterminée par le repère mobile $\left\{X, \sigma_{1}, \sigma_{2}\right\}$.

Alors, on a

$$
L P\left(\mathscr{F}_{u}\right)\left(\left[S^{3}\right]\right)=2+\{(1+u) /(1-u)\}+\{(1-u) /(1+u)\} .
$$

Nous démontrons (7.1).

Soient $F_{1}, F_{2}$ les orbites du flot $\phi_{t}^{u}$ qui passent par les points

$$
g_{1}=\left(\begin{array}{ll}
1 & 0 \\
0 & 1
\end{array}\right), \quad g_{2}=\left(\begin{array}{rr}
0 & 1 \\
-1 & 0
\end{array}\right)
$$

respectivement. Alors, l'orbite $F_{1}$ (resp. $F_{2}$ ) est une orbite périodique dont la période est $p_{1}=2 \pi /(1+u)$ (resp. $p_{2}=2 \pi /(1-u)$ ) pour tous $u$.

Fixons $u$ et considérons les applications tangentes suivantes

$$
H_{i}=\left(\phi_{p_{i}}^{u}\right)_{*}: T_{g_{i}}(S U(2)) \longrightarrow T_{g_{i}}(S U(2))
$$

pour $i=1,2$. Posons un élément $Z$ de $T_{g_{1}}(S U(2))$ (resp. $T_{g_{2}}(S U(2))$ )

$$
Z=x \sigma_{1}+y \sigma_{2}=\left(\begin{array}{rr}
0 & -\bar{z} \\
z & 0
\end{array}\right)
$$

où $z=x+y \sqrt{-1}$. Alors, on a

$$
H_{i}(Z)=\left(\begin{array}{cc}
0 & -\bar{z}_{i} \\
z_{i} & 0
\end{array}\right) \quad \text { pour } i=1,2
$$

où

$$
z_{1}=z \cdot \exp (2 \pi \sqrt{-1}(1-\mathrm{u}) /(1+\mathrm{u}))\left(\operatorname{resp} . z_{2}=z \cdot \exp (2 \pi \sqrt{-1}(1+\mathrm{u}) /(1-\mathrm{u}))\right) .
$$

Et on sait que la métrique quasi-fibrée de $\nu\left(\mathscr{F}_{u}\right)$ est induité d'une unique métrique bi-invariante de $S U(2)$ telle que $\left\{X, \sigma_{1}, \sigma_{2}\right\}$ est un repère orthonormé. Donc, l'holonomie linéaire de la feuille $F_{1}$ (resp. $F_{2}$ ) orientée par le flot $\phi_{t}^{u}$ est $\exp (2 \pi \sqrt{-1}(1-u) /(1+u))(\operatorname{resp} . \exp (2 \pi \sqrt{-1}(1+u) /(1-u)))(\in S O(2))$.

En plus, il est claire que les feuilletages $\mathscr{F}_{u}$ admettent une 1-extension singulière $(\mathscr{H}, \Sigma)$ telle que $\Sigma=F_{1} \cup F_{2}$ et les $t$-indices sont 1 .

Par suite, on déduit immédiatement du théorème 3.3:

$$
L P\left(\mathscr{F}_{u}\right)\left(\left[S^{3}\right]\right) \equiv\{(1-u) /(1+u)\}+\{(1+u) /(1-u)\} \quad \bmod Z .
$$

D'autre part, on remarque que les feuilles du feuilletage $\mathscr{F}_{0}$ sont les fibres d'une fibration en cercle sur $S^{2}$. Alors, il n'est pas difficile de voir que les invariants orientés du flot $\phi_{t}^{0}$ est $\{1 ;(0,0,0,0)\}$. Donc, par les lemmes 4.3 et 4.4 , on a 


$$
L P\left(\mathscr{F}_{0}\right)\left(\left[S^{3}\right]\right)=4 .
$$

Maintenant, si on remarque que la fonction $L P\left(\mathscr{F}_{u}\right)\left(\left[S^{3}\right]\right)$ est différentiable en $u$, on a (7.1) par (7.2) et (7.3).

\title{
Références
}

[1] P. Baum and R. Bott, Singularities of Holomorphic Foliations, J. Differential Geometry, 7 (1972), 279-342.

[2] R. Blumenthal, Transversely homogeneous foliations, Ann. Inst. Fourier (Grenoble), 29 (1979), 143-158.

[ 3 ] Y. Clifton and S. Wolfgang, The Euler class as an obstraction in the theory of foliations, Proc. Nat. Acad. Sci., 50 (1963), 949-959.

[4] D. B. A. Epstein, Periodic flows on three-manifolds, Ann. of Math., 95 (1972), 66-82.

[5] F. Fuller, An index of fixed-point type for perodic orbits, Amer. J. Math., 89 (1967), 133-148.

[6] W. Greub, S. Halperin and R. Vanstone, Connections, Curvature, and Cohomology; vol. III, Academic press, 1972.

[ 7 ] A. Haefliger, Variétés feuilletées, Ann. Scoula Norm. Sup., Pisa, 3 (1962), 367397.

[ 8 ] R. Hermann, On the differential geometry of foliations, Ann. of Math., 72 (1960), 445-457.

[9] M. Hirsch, Stability of Compact Leaves of Foliation, Dynamical Systems, Academic press, 1971, 135-153.

[10] F. W. Kamber and P. Tondeur, Foliated Bundles and Characteristic Classes, Lecture Notes in Math., 493, Springer-Verlag, 1975.

[11] S. Kobayashi, Transformation groups in differential geometry, Springer-Verlag, 1972.

[12] C. Lazarov and J. Pasternack, Secondary characteristic classes for riemannian foliations, J. Differential Geometry, 11 (1976), 365-385.

[13] J. Milnor, Topology from the differentiable view point, The University press of Virginia, 1969.

[14] P. Molino, Etude des feuilletages transversalement complets et applications, Ann. Sci. École Norm. Sup., 10 (1977), 289-307.

[15] P. Orlik, Seifert Manifolds, Lecture Notes in Math., 291, Springer-Verlag, 1972.

[16] P. Orlik and F. Raymond, Actions of SO (2) on 3-manifolds, Proceedings of the conference on Transformation Groups, Springer-Verlag, 1968, 297-318.

[17] K. Yamato, Sur la classe caractéristique exotique de Lazarov-Pasternack en codimension 2, I, C. R. Acad. Sci. Paris, 289 (1979), Série A, 537-540.

\author{
Département de Mathématique \\ UNIVERSITÉ D'OSAKA \\ TOYONAKA, OSAKA, 560 \\ JAPON
}

